ARTICLE

DOI: $10.1038 / s 41467-018-06145-w$

\title{
A conserved glycine harboring disease-associated mutations permits NMDA receptor slow deactivation and high $\mathrm{Ca}^{2+}$ permeability
}

\author{
Johansen B. Amin 1,2,3, Xiaoling Leng ${ }^{4}$, Aaron Gochman ${ }^{5}$, Huan-Xiang Zhou ${ }^{4,6}$ \& Lonnie P. Wollmuth (1) 3,5,7
}

A variety of de novo and inherited missense mutations associated with neurological disorders are found in the NMDA receptor M4 transmembrane helices, which are peripheral to the pore domain in eukaryotic ionotropic glutamate receptors. Subsets of these mutations affect receptor gating with dramatic effects, including in one instance halting it, occurring at a conserved glycine near the extracellular end of M4. Functional experiments and molecular dynamic simulations of constructs with and without substitutions at this glycine indicate that it acts as a hinge, permitting the intracellular portion of the ion channel to laterally expand. This expansion stabilizes long-lived open states leading to slow deactivation and high $\mathrm{Ca}^{2+}$ permeability. Our studies provide a functional and structural framework for the effect of missense mutations on NMDARs at central synapses and highlight how the M4 segment may represent a pathway for intracellular modulation of NMDA receptor function.

\footnotetext{
${ }^{1}$ Graduate Program in Cellular and Molecular Pharmacology, Stony Brook University, Stony Brook, NY 11794-5230, USA. ${ }^{2}$ Medical Scientist Training Program (MSTP), Stony Brook University, Stony Brook, NY 11794-5230, USA. ${ }^{3}$ Center for Nervous System Disorders, Stony Brook University, Stony Brook, NY $11794-$ 5230, USA. ${ }^{4}$ Department of Physics and Institute of Molecular Biophysics, Florida State University, Tallahassee, FL 32306, USA. ${ }^{5}$ Department of Neurobiology and Behavior, Stony Brook University, Stony Brook, NY 11794-5230, USA. ${ }^{6}$ Departments of Chemistry and Physics, University of Illinois at Chicago, Chicago, IL 60607, USA. ${ }^{7}$ Department of Biochemistry and Cell Biology, Stony Brook University, Stony Brook, NY 11794-5230, USA.

Correspondence and requests for materials should be addressed to L.P.W. (email: lonnie.wollmuth@stonybrook.edu)
} 
A berrant ion channel functions, or channelopathies, are a major cause of neurological disorders ${ }^{1}$. Ionotropic glutamate receptors (iGluRs), including AMPA (AMPAR) and NMDA (NMDAR) receptor subtypes, are glutamate-gated ion channels found throughout the brain and participate in almost all brain functions. In terms of channelopathies, iGluR autoantibodies are associated with paraneoplastic encephalopathies and the neurological dysfunction in neuropsychiatric lupus ${ }^{2,3}$. Further, numerous de novo and inherited missense mutations have been identified in iGluR subunits that are associated with a wide spectrum of disorders, including epilepsy, intellectual disability, movement disorders, and schizophrenia ${ }^{4-7}$.

iGluRs are highly modular proteins composed of four domains (Fig. 1a) and function as tetrameric complexes ${ }^{8}$. Interestingly, missense mutations in the ligand-binding domain (LBD) and transmembrane domain (TMD) forming the ion channel are typically associated with more severe clinical phenotypes ${ }^{7,9}$, reflecting their central role in agonist binding and ion channel opening. The ion channel core is formed by two transmembrane helices, M1 and M3, and an intracellular reentrant pore helix, $\mathrm{M} 2^{10,11}$. In eukaryotic iGluRs, there is an additional transmembrane helix, M4, that packs against the M1 and M3 helices of a neighboring subunit. The M4 helix plays an important role in receptor biogenesis and ion channel function ${ }^{12-15}$

In the NMDAR M4 segments, there are numerous missense mutations associated with clinical pathologies (Fig. 1b, c; Supplementary Table 1). These mutations are primarily located in the extreme extracellular third of the M4 segments (Fig. 1c), which participates strongly in ion channel gating ${ }^{16,17}$, implicating gating deficits as a potential cause for these disease phenotypes.

Here we address the contribution of these M4 missense mutations to receptor function. Although many of these mutations impact receptor gating, the most notable and robust effects occur at a highly conserved glycine (Supplementary Figure 1) positioned near the extracellular end of the M4 segments. Missense mutations at this position in the GluN1 and GluN2B subunits have been identified in multiple patients, and the resulting changes in side chain properties can be either great (glycine-to-
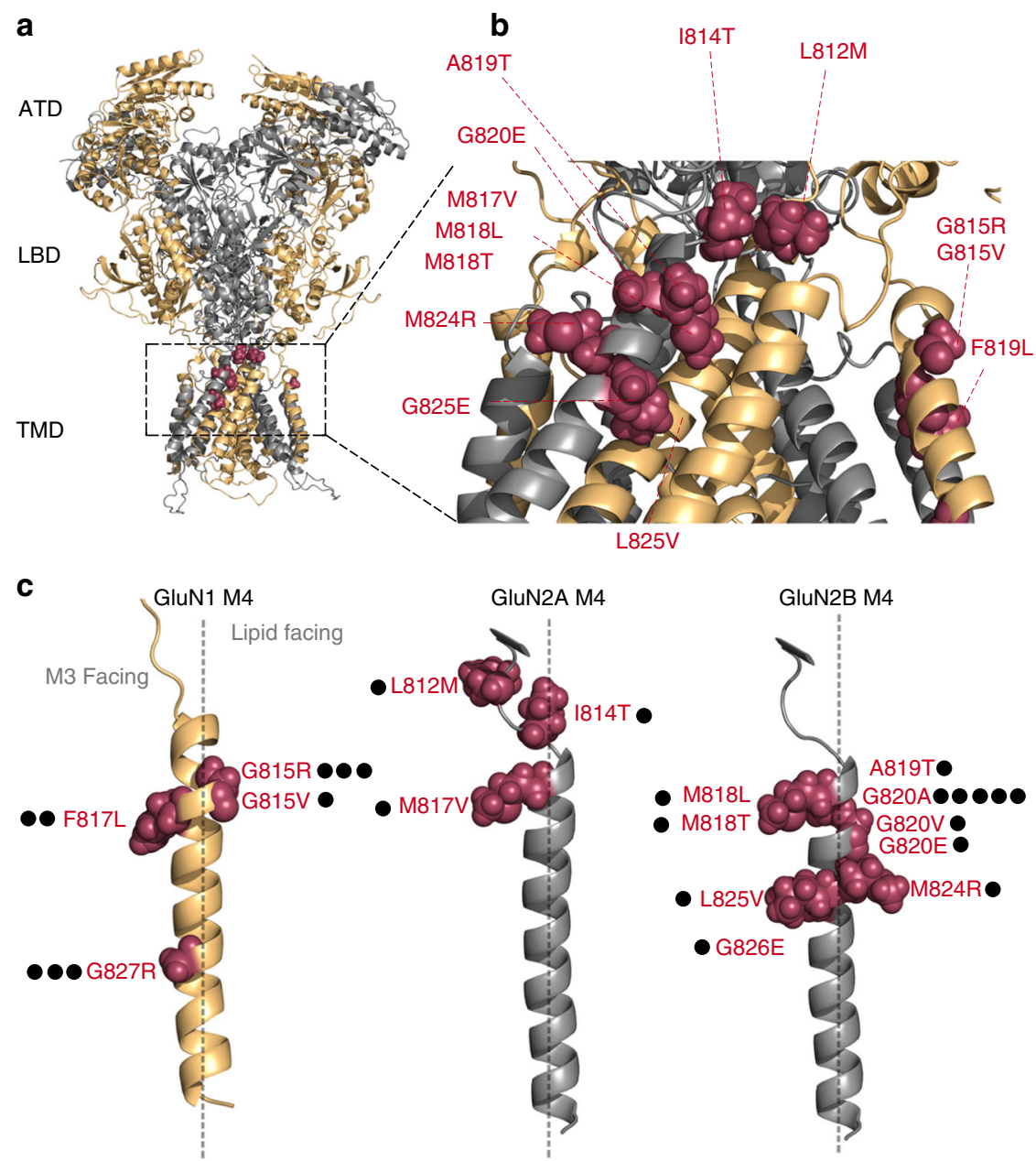

Fig. 1 Topology of NMDA receptors and distribution of missense mutations in and around the M4 transmembrane segments. a Model NMDAR structure (structure based on 4TLM of GluN1/GluN2B) ${ }^{16}$ lacking the intracellular C-terminal domain (CTD). Subunits are colored light orange (GluN1) and gray 60\% (GluN2). For iGluRs, the tetrameric complex is composed of four highly modular domains: the extracellularly located amino-terminal (ATD) and ligandbinding (LBD) domains; the membrane-spanning transmembrane domain (TMD) forming the ion channel; and the CTD. Positions highlighted in magenta are disease-associated missense mutations in the GluN1, GluN2A, or GluN2B M4 transmembrane segment or the S2-M4 linker (L812M and I814T in GluN2A). $\mathbf{b}$ An enlarged view of the extracellular end of the TMD highlighting the distribution of missense mutations in and around the M4 segments. c The GluN1, GluN2A, and GluN2B M4 segments indicting the specific location of missense mutations. Each dot represents a patient in which the mutation has been identified (Supplementary Table 1). The dashed line separates M3 (leftside) versus lipid (rightside) facing portions of the M4 transmembrane segment 
a

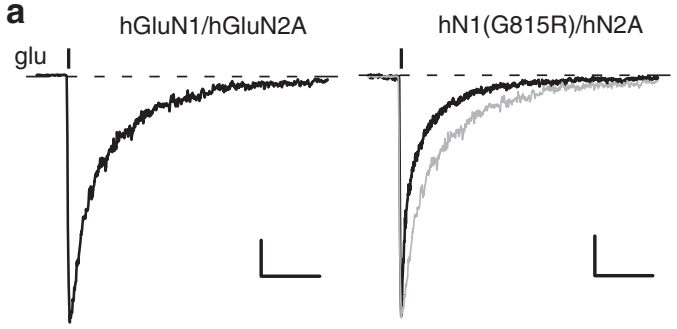

c hGluN1/hGlun2B

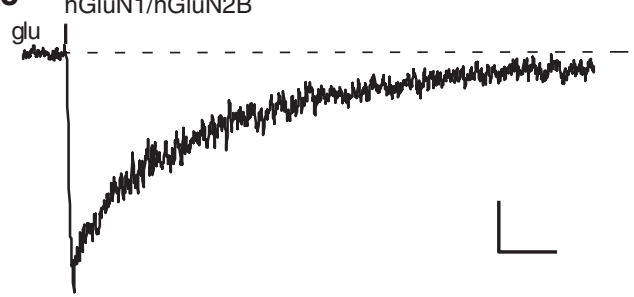

$\mathrm{hN} 1 / \mathrm{hN} 2 \mathrm{~B}(\mathrm{G} 820 \mathrm{~A})$

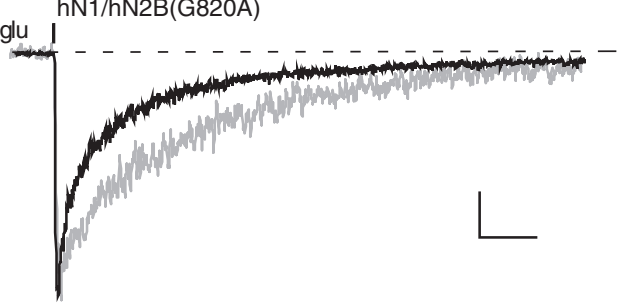

b
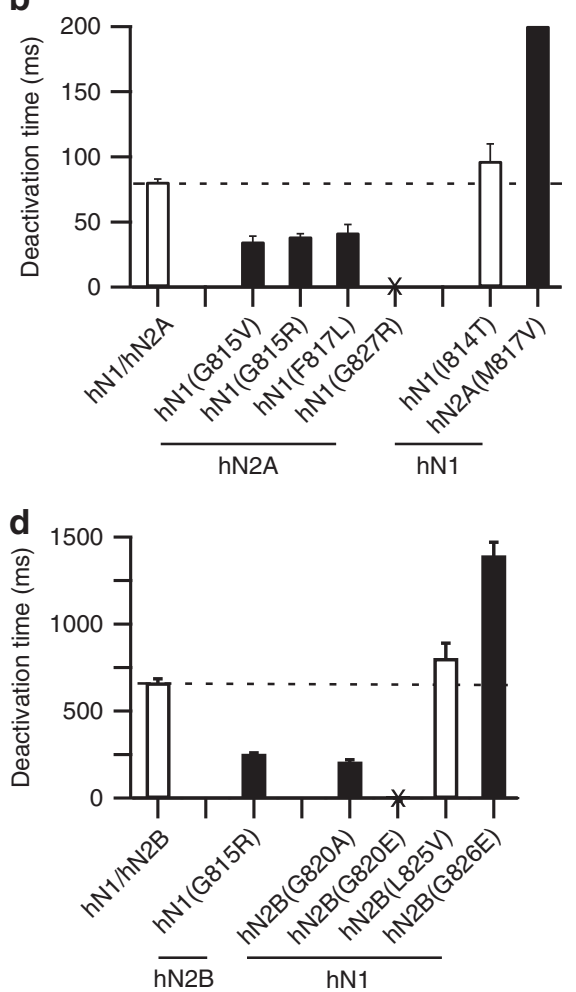

Fig. 2 Missense mutations in M4 segments alter NMDAR gating. a, c Whole-cell currents from HEK293 cells expressing human NMDAR subunits, either hGluN1/hGluN2A (a) or hGluN1/GluN2B (c) or the same receptor with a missense mutation at the conserved glycine. Currents were elicited by a 2 ms application of glutamate $(1 \mathrm{mM})$ in the continuous presence of glycine $(0.1 \mathrm{mM})$, as approximately occurs at synapses. Gray traces are wild type. Holding potential, $-70 \mathrm{mV}$. Scale bars: (a) $100 \mathrm{pA}$, wild type; $25 \mathrm{pA}$, hN1(G815R); (c) $50 \mathrm{pA}$; time base was $0.2 \mathrm{~s}$ for all. b, d Bar graphs (mean \pm SEM) ( $n>5$ ) showing deactivation rates for hGluN1/hGluN2A (b) or hGluN1/hGluN2B (d) (Supplementary Table 2). No whole-cell current could be detected for hGluN1 (G827R)/hGluN2A or hGluN1/hGluN2B(G820E), which were tested on at least three different transfection cycles (Supplementary Figure 2). Solid bars indicate values significantly different from wild type $(p<0.05, t$-test $)$

arginine) or subtle (glycine-to-alanine). For both NMDAR subunits, but most notably the obligatory GuN1 subunit, mutations at this conserved glycine drastically impair the stability of longlived open states, greatly enhancing the rate of receptor deactivation as well as attenuating $\mathrm{Ca}^{2+}$ permeability. Molecular dynamics simulations show that this glycine acts as a hinge, allowing for the C-terminal portion of the GluN1 M4 segment and the ion channel core to laterally expand. These results have strong implications for how neurological disorders are caused at the ion channel level and implicate the M4 as a major conduit for modulation of ion channel function.

\section{Results}

Disease-associated mutations are prominent at glycines. At present, 23 patients with neurological disorders have been identified with de novo missense mutations in the M4 segments of NMDAR subunits (Fig. 1). The clinical phenotype for thesepatients is typically severe, encompassing epileptic encephalopathies and intellectual disabilities (Supplementary Table 1). For the majority of patients, the mutations occur at glycines (15 out of 23 patients). In transmembrane regions, the most common missense mutation associated with disease is glycine-toarginine ${ }^{18,19}$. Glycines often participate in critical structural roles in transmembrane segments, including acting as a notch for transmembrane segment interactions ${ }^{20}$ and to provide flexibility to transmembrane segments ${ }^{21}$. Substitution of a charged side chain for a glycine can strongly disrupt these functions.
Of the 15 patients with missense mutations at glycines, eight carry mutations to charged side chains: six are arginines (R) [GluN1(G815R), GluN1(G827R)] and two are glutamate (E) [GluN2B(G820E), GluN2B(G826E)]. However, for a glycine at the external end of M4, a number of patients have mutations that have less dramatic changes in the nature of the side chain, from glycine to alanine (A) or valine (V). This glycine, G815 in GluN1, G819 in GluN2A, and G820 in GluN2B, is one of only three positions completely conserved across all mammalian iGluR M4 segments (Supplementary Figure 1a) and also shows considerable conservation across many different species (Supplementary Figure 1b). That less dramatic missense mutations (e.g., alanine) can have a clinical phenotype and its conservation suggest that this glycine plays an important role in iGluR function.

M4 missense mutations dramatically alter receptor gating. The association of NMDAR missense mutations with neurological diseases could reflect alterations in the rules of NMDAR subunit assembly, trafficking ${ }^{22}$, interaction with other intracellular or extracellular proteins, and/or receptor functional properties, such as gating, ion permeation, or channel block ${ }^{23,24}$. The majority of missense mutations in the M4 segment are positioned at the external ends, which participate strongly in gating and make no apparent contribution to a cell biological function (e.g., assembly) 16. We therefore characterized the effect of many of these missense mutations on receptor gating (Fig. 2 and Supplementary Figure 2) using an external solution that approximates physiological conditions (see Methods). 
a
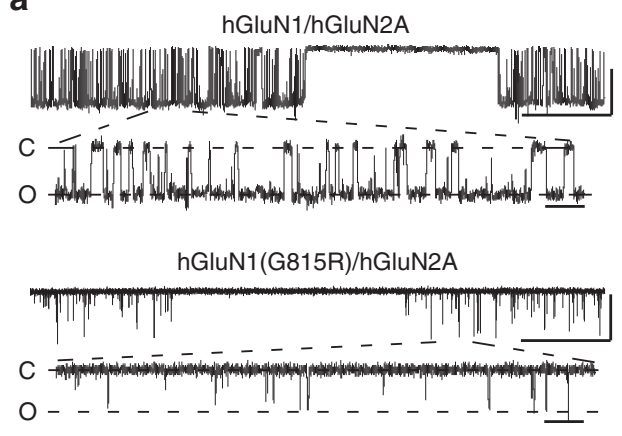

C
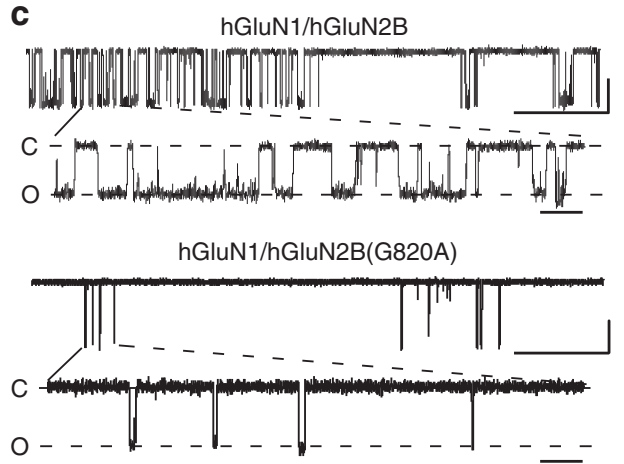

b

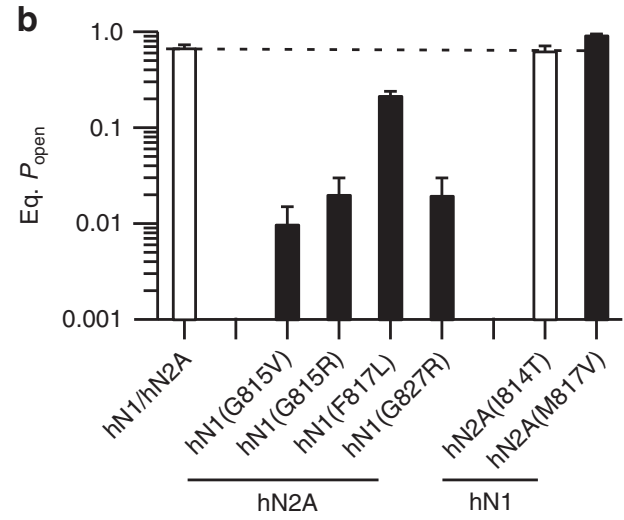

d

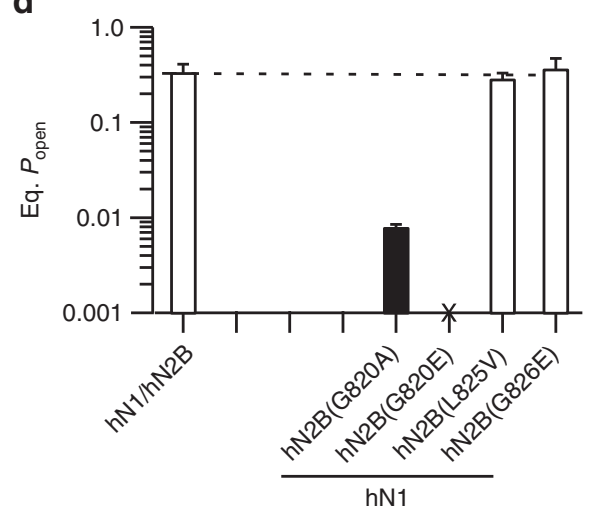

Fig. 3 Single channel recordings of missense mutations in M4 segments. a, c Example single channel recordings of hGluN1/hGluN2A (a) or hGluN1/ hGluN2B (c) or the same receptor containing missense mutations at the conserved glycine. Recordings were performed in the on-cell configuration (holding potential, $+100 \mathrm{mV}$ ). Downward deflections are inward currents. For each construct, the top half shows a low-resolution (filtered at $1 \mathrm{kHz}$ ) and the bottom half a higher resolution portion of same record ( $3 \mathrm{kHz}$ ). Scale bars: $5 \mathrm{pAs}$ for all; time base is $500 \mathrm{~ms}$ (upper trace for each construct) and $20 \mathrm{~ms}$ (lower trace for each construct). b, d Equilibrium open probability (eq. $P_{\text {open }}$ ) (mean \pm SEM) ( $n>4$ patches) for hGluN1/hGluN2A (b) or hGluN1/hGluN2B (d) (Supplementary Table 3). Solid bars indicate values significantly different from wild type ( $p<0.05$, $t$-test). For GluN1/GluN2B(G820E), we could not detect glutamate-activated current either in on-cell patches or whole-cell mode

A critical determinant of synaptic NMDARs is their slow deactivation time course, which is GluN2-subunit specific ${ }^{25-27}$. Currents in human GluN1/GluN2A (hN1/hN2A) (Fig. 2a, left) and GluN1/GluN2B (hN1/hN2B) (Fig. 2c, top), induced by a brief glutamate application as occurs at synapses, decay slowly with the time courses best fit by double exponentials (weighted $\tau \mathrm{s}$, $81 \pm 2 \mathrm{~ms}, n=11$ and $660 \pm 30 \mathrm{~ms}, n=8$, respectively) (mean \pm SEM, $n=$ number of recordings) (Fig. 2b, d; Supplementary Table 2). As expected, the decay was much slower for GluN2Bcontaining receptors ${ }^{26}$.

Several of the missense mutations had no significant effect on deactivation times (Fig. 2b, d, open bars), whereas two, hGluN2A $(\mathrm{M} 817 \mathrm{~V})^{17}$ and hGluN2B(G826E), significantly slowed deactivation. However, the most prominent effects of the M4 missense mutations was a significant speeding of deactivation, which was notable for mutations at the conserved glycine in hGluN1, G815V $(35 \pm 4 \mathrm{~ms}, n=5)$ and G815R $(39 \pm 2 \mathrm{~ms}, n=6)$ (Fig. 2b, filled bars) and in hGluN2B, G820A (205.2 $\pm 12 \mathrm{~ms}, n=7$ ) (Fig. 2d, filled bars). hGluN1(G815R) reduced the deactivation times by about half whether in the hGluN2A or hGluN2B background. We could not detect glutamate-activated whole-cell current for hGluN1(G827R)/hGluN2A or hGluN1/hGluN2B(G820E), either with brief or more sustained (Supplementary Figure 2) applications.

Although, the missense mutations could have additional effects on receptor function (e.g., permeation) or cell biology, these strong effects on receptor deactivation (Fig. 2) as well as those on desensitization (Supplementary Figure 2), presumably contribute to their associated clinical phenotype. Here we will focus on how these mutations impact receptor function.

Subsets of missense mutations strongly alter single channel activity. To begin to understand how the missense mutations impact receptor gating, we recorded single channel activity in the on-cell mode (Fig. 3a-d; Supplementary Figure 3 and Supplementary Table 3). To obtain more mechanistic insight, we used an external solution that optimizes NMDAR activity (see Methods). Wild-type hGluN1/hGluN2A showed a mean equilibrium open probability (eq. $\left.P_{\text {open }}\right)$ of $0.69 \pm 0.04, n=6($ mean \pm SEM, $n=$ number of single channel patches) (Fig. $3 \mathrm{a}, \mathrm{c}$ ) whereas hGluN1/hGluN2B showed a reduced eq. $P_{\text {open }}(0.34 \pm 0.07, n=5)$ (Fig. 3b, d, Supplementary Table 3), as found previously 28,29 . Certain missense mutations had no effect on activity (Fig. 3b, d, open bars), whereas one, GluN2A(M817V), showed a significant potentiation ${ }^{17}$.

On the other hand, most tested missense mutations reduced single channel activity with the strongest effects occurring at glycines. At the conserved glycine, G815 in GluN1 and G820 in GluN2B, eq. $P_{\text {open }}$ was $<0.02$ (G815V, $0.01 \pm 0.005, n=4$; G815R, $0.02 \pm 0.01, n=5$; and G820A, $0.008 \pm 0.0005, n=4$ ). For GluN1/GluN2B(G820E), which showed no whole-cell 

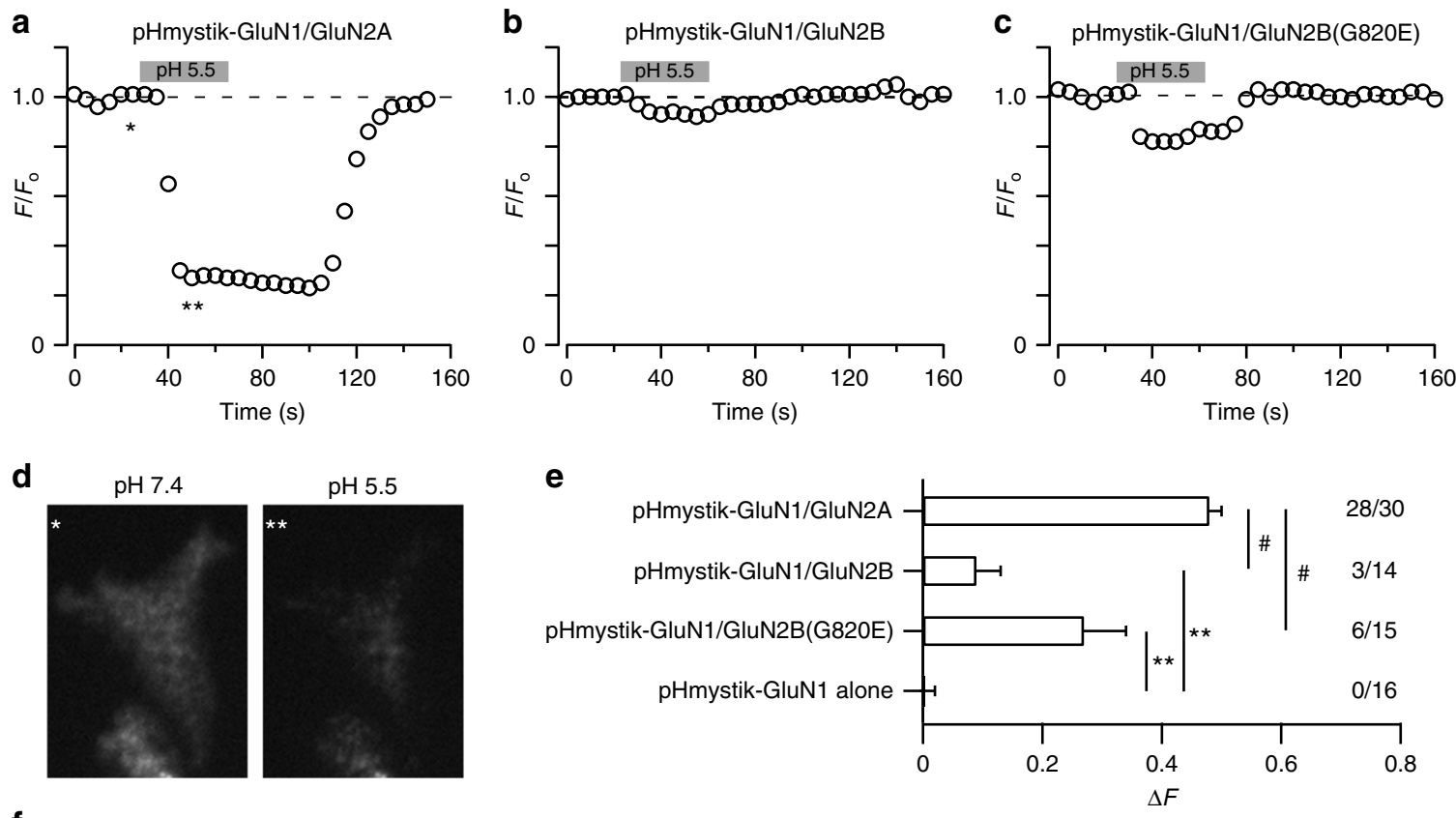

f

\section{Wild type} GluN1/GluN2A

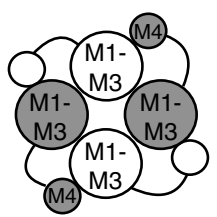

g Triheteromeric One mutation

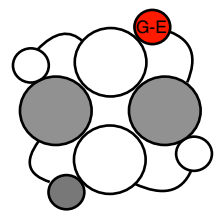

h

Diheteromeric Two mutations

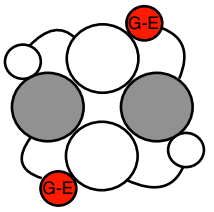

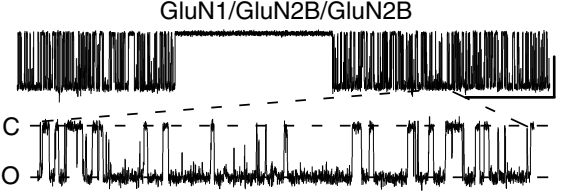

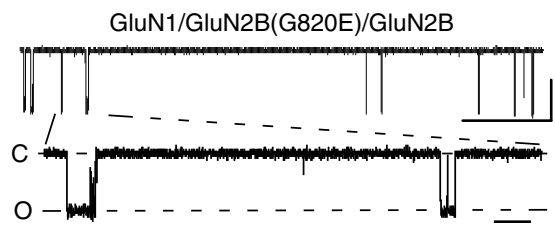

GluN1/GluN2B(G820E)/GluN2B(G820E) no detectable membrane current

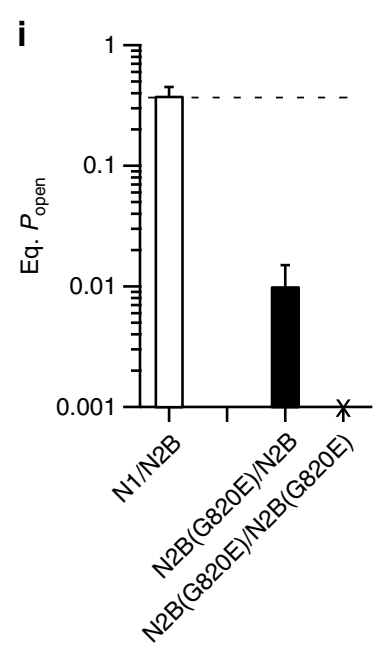

Fig. 4 The diheteromeric missense mutation of a conserved glycine is pore dead. a-c Assaying surface expression using a pH-sensitive GFP16 indicates that GluN1/GluN2B(G820E) is expressed on the membrane. GFP intensity as the extracellular solution pH was changed from 7.4 to 5.5 (gray bar, $30 \mathrm{~s}$ ) and back again. $\mathbf{d}$ Representative cell images are from the time-points labeled in a with asterisks. Image $\mathrm{pH} 7.4$ (single asterisk) is baseline fluorescence $\left(F_{0}\right)$, whereas image $\mathrm{pH} 5.5$ (double asterisks) is test fluorescence $\left(F_{\text {test }}\right)$. The change in fluorescence $\left(\Delta F=F_{\mathrm{o}}-F_{\text {test }}\right)$ was used as an index of surface expression. Sampling rate, $5 \mathrm{~s}$. The strong fluorescent background for GluN2B constructs presumably reflects subunits trapped in endoplasmic reticulum. e Changes in cell fluorescence at low pH $(\Delta F)$. Significant differences from pHmystick-GluN1/GluN2A and pHmystik-GluN1 alone are indicated by \# and **, respectively, ( $p<0.05, t$-test). The numbers (far right) indicate the number of cells that showed detectable changes in fluorescence relative to the total number of cells tested. $\mathbf{f}-\mathbf{h}$ Single channel recordings of NMDAR constructs containing the coiled-coiled domain in C-terminus ${ }^{30}$, either wild type (f), the triheteromeric containing a single copy of G820E ( $\mathbf{g}$ ) or the diheteromeric containing 2 copies of G820E (h), which does not show detectable glutamateactivated current (Figs. 2 and 3), though it expresses on the membrane. The triheteromeric receptor shows detectable single channel activity. Scale bars: $5 \mathrm{pAs}$ for all; time base is $500 \mathrm{~ms}$ (upper trace for each construct) and $20 \mathrm{~ms}$ (lower trace for each construct). i Mean ( \pm SEM) showing eq. $P_{\text {open }}$ for wild type $(n=4)$ and triheteromeric GluN1/GluN2B/GluN2B(G820E) $(n=3)$

current amplitudes, no single channel activity could be detected (Fig. 3d; Supplementary Table 3). We detected single channe1 activity for a missense mutation at a glycine positioned more intracellularly, GluN1(G827R), for which we could not detect whole-cell current. Again, like other missense mutations at glycines, gating in GluN1(G827R) was strongly disrupted $(0.02 \pm 0.01, n=4)$ (Fig. 3b). The lack of detectable whole-cell currents for GluN1(G827R) probably reflects that C-terminal positions in M4, especially this glycine, impact receptor surface expression $^{16}$.

A missense mutation at the conserved glycine is pore dead. The lack of glutamate-activated current for GluN2B(G820E), either in 


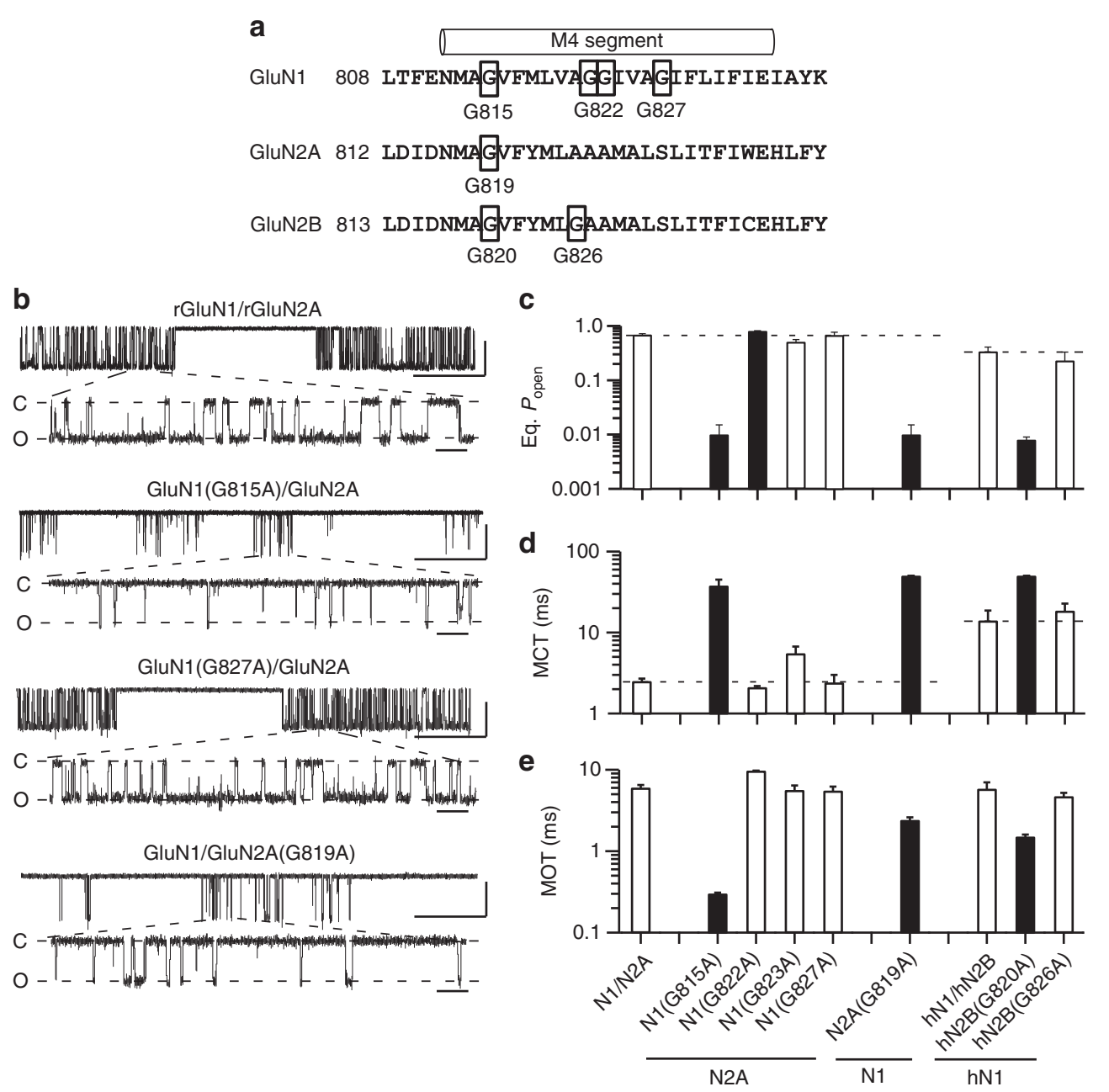

Fig. 5 Alanine substitutions of glycine in the M4 segments reveal that the conserved glycine has a unique functional role in gating. a Sequence of M4 segments and adjacent regions highlighting glycines. b Example single channel recordings in the on-cell configuration of wild type rat GluN1/GluN2A and various receptors containing glycine-to-alanine substitutions. Recordings displayed as in Fig. 3a. Scale bars: 5 pAs for all; time base is 500 ms (upper trace for each construct) and 20 ms (lower trace for each construct). c-e Mean \pm SEM ( $n>4$ patches) showing equilibrium open probability (eq. $P_{\text {open }}$ ) (c), mean closed time (MCT) (d), and mean open time (MOT) (e) for alanine substitutions of glycines in the M4 segments of rat GluN1 and GluN2A and human GluN1 (hGluN1) and GluN2B (hGluN2B) (Supplementary Table 4). Solid bars indicate values significantly different from their respective wild types $(p<0.01, t$-test)

the whole-cell or on-cell configuration (Figs. 2 and 3), could reflect disrupted cell biology, either assembly and/or trafficking, or a complete lack of gating. To test these alternatives, we measured surface expression of GluN2B(G820E) using pHmystik, a $\mathrm{pH}$-sensitive GFP, and TIRF microscopy ${ }^{16}$. In contrast to wildtype GluN1/GluN2A (Fig. 4a), wild-type GluN1/GluN2B showed poor surface expression (Fig. 4b, e) with the strong background fluorescence presumably due to receptors trapped in the endoplasmic reticulum. In contrast, GluN1/GluN2B(G820E) showed robust surface expression (Fig. 4c, e). Thus, this missense mutation in GluN2B, G820E, at least in a diheteromeric form, is pore dead: it can get to the membrane surface but cannot gate, an effect found for other positions at the extracellular end of GluN2 M4 ${ }^{16}$.

To further test the importance of the conserved glycine in GluN2B, we performed a rescue experiment in which we recorded single channel activity from triheteromeric receptors, where the receptor contains a single copy of G820E (Fig. $4 \mathrm{f}-\mathrm{i}$ ). To generate triheteromeric receptors, we used NMDAR constructs having coiled-coiled domains ${ }^{30}$ that permit only specific subunit combinations to reach the plasma membrane. The triheteromeric form, GluN1/GluN2B/GluN2B(G820E), is functional (Fig. 4g), but like other missense mutations at this conserved glycine, gating is severely restricted (eq. $P_{\text {open }}, 0.007 \pm 0.001, n=3$ ) (Fig. $4 \mathrm{i}$ ).

In summary, many of the missense mutations in the M4 segment strongly restrict NMDAR gating (Figs. 2, 3 and 4; Supplementary Figure 3). These effects are notable at glycines, but are most severe at a conserved glycine, leading to in the diheteromeric form a receptor that transits to the membrane but is unable to gate (Fig. $4 \mathrm{a}-\mathrm{e}$ ).

The conserved glycine in the M4s is key for pore opening. The M4 segments must be displaced for efficient pore opening to occur ${ }^{31}$. One possible explanation for the effect of missense mutations at glycines in the various M4 segments is that these glycines provide local flexibility for the gating process to proceed. To test this possibility, we substituted each individual glycine in the M4 segments of GluN1, GluN2A, or GluN2B (Fig. 5a), including those not associated with missense mutations, with alanine, which would constrain local flexibility ${ }^{32}$ while not greatly altering local interactions as might occur with charged side chains. We measured single-channel activity for these constructs (Fig. 5b-e). 

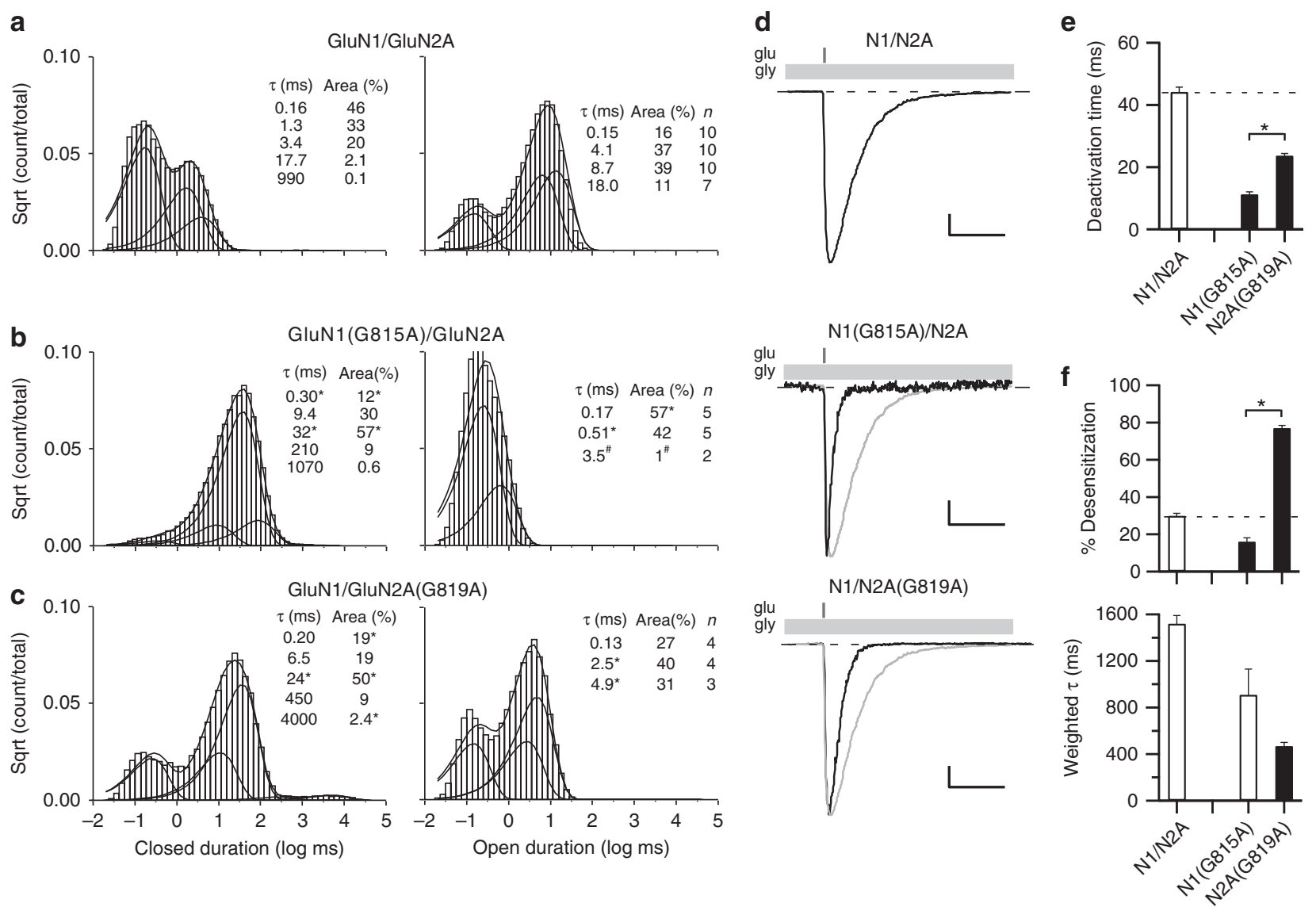

Fig. 6 Constraining the conserved glycine prevents entry into long-lived open states and speeds deactivation. a-c Closed (left) and open (right) time histograms for wild type (a) or for receptors containing an alanine substitution at the conserved glycine in GluN1 (b) or GluN2A (c). All closed time histograms were best fit with 5 exponentials (Supplementary Table 5), whereas open time histograms were best fit typically with 4 (wild type), 2 (GluN1 (G815A)), or 3 (GluN2A(G819A)) exponentials (Supplementary Table 6) (see Methods). Smooth lines are associated exponential fits. Insets, mean closed and open state durations ( $\tau, \mathrm{ms})$, occupancies $(\alpha, \%)$, and for open time distributions how many recordings contained that open time (Supplementary Tables 5 \& 6). For GluN1(G815A), the longest-lived open state ( $3.5 \mathrm{~ms}$ ) was identified in only 2 out of 5 recordings; \# indicates that we did not do statistics on this state. $\mathbf{d}$ Whole-cell currents in response to a $2 \mathrm{~ms}$ application of glutamate $(1 \mathrm{mM}$, gray bars) applied in the continuous presence of glycine (light gray bars). Recordings were made and analyzed as in Fig. 2a but included extracellular $0.05 \mathrm{mM}$ EDTA to remove effects of $Z \mathrm{n}^{2+}$ as done for single channel recordings. Scale bars: 300 pA, wild type; 10 pA, N1(G815A); 100 pA, N2A(G819A); time base is 100 ms for all. e, f Mean \pm SEM ( $n>5$ ) showing deactivation rates (e) or desensitization properties (f) for constructs shown in $\mathbf{d}$. Solid bars indicate values significantly different from wild type, whereas asterisks indicate values different from each other $(p<0.05$, ANOVA,Tukey)

Many of the glycine-to-alanine substitutions in GluN1, GluN2A, or GluN2B M4s either had no effect (open bars) or weakly altered eq. $P_{\text {open }}$ (G822 in GluN1). Notably, a charged missense mutation in a GluN1 glycine (G827R) had a dramatic effect on gating (Fig. 3b), but the subtler alanine had no significant effect (Fig. 5c; Supplementary Table 4). In contrast, alanine substitutions at the conserved glycine in all three subunits, GluN1(G815A), GluN2A(G819A), and hGluN2B (G820A), dramatically altered gating, reducing eq. $P_{\text {open }}$ to less than 0.01 . Thus, the conserved glycine plays a unique role irreplaceable by any other side chain.

Alanine substitutions at the conserved glycines dramatically reduced receptor gating by significantly altering mean closed time (MCT) and mean open time (MOT) (Fig. 5d, e; Supplementary Table 4). In addition, the alanine substitution in GluN1 reduced single-channel activity to a similar extent as the charged (G815R) missense mutation (Fig. 3b) or when tryptophan was substituted ${ }^{16}$.

The conserved glycine permits slow deactivation. To further address the impact of the conserved glycines to gating, we characterized closed and open time distributions of the glycineto-alanine substitutions in GluN1 and GluN2A (Fig. 6a-c). We focused functional experiments on GluN2A rather than GluN2B because of its more robust eq. $P_{\text {open }}$.

Our wild-type recordings were best described by 5 closed states (Fig. 6a, left; Supplementary Table 5) ${ }^{33}$, as were the recordings for alanine substitutions at the conserved glycine position in GluN1 (Fig. 6b, left) and GluN2A (Fig. 6c, left). Their time course and specific duration, however, showed significant variation (Supplementary Table 5).

Wild-type GluN1/GluN2A shows modal gating consisting of a brief $\left(\mathrm{O}_{1}\right)$ and three longer lived $\left(\mathrm{O}_{2}, \mathrm{O}_{3}\right.$, and $\left.\mathrm{O}_{4}\right)$ open states ${ }^{28}$. For our recordings, open time distributions of wild type were also generally best described by 4 open states (Fig. 6a, right; Supplementary Table 6). In contrast, the open time distributions for glycine-to-alanine substitutions were at best only fit by 3 components, with a long-lived open state completely absent (Fig. 6b, c, right). Further, while GluN1(G815A) and GluN2A (G819A) visited a third open state, the durations were shorter ( $3.5 \mathrm{~ms}, n=2$ and $4.9 \pm 1.0 \mathrm{~ms}, n=3$, respectively, compared to $8.7 \pm 0.6 \mathrm{~ms}, n=10$ for wild type). Finally, GluN1(G815A) visited 
a third open state extremely rarely ( 2 out of 5 recordings), around $1 \%$ of the time compared to $39 \pm 10 \%$ for wild type, highlighting the more prominent role of the GluN1 conserved glycine.

The kinetics of the NMDAR single-channel activity mediate the slow macroscopic deactivation time courses characteristic of synaptic NMDARs ${ }^{25,34}$. We therefore directly compared deactivation rates for GluN1/GluN2A, GluN1(G815A)/GluN2A, and GluN1/GluN2A(G819A) under conditions comparable to those done for single channel recordings (extracellular $0.05 \mathrm{mM}$ EDTA) (Fig. 6d). Consistent with the reduced long open times, the deactivation times for GluN1(G815A) $(11.3 \pm 0.8 \mathrm{~ms}, n=5)$ and GluN2A(G819A) $(23.7 \pm 0.7 \mathrm{~ms}, n=5)$ were significantly faster than that for wild type $(44.2 \pm 1.5 \mathrm{~ms}, n=5)$. Paralleling the results for MOTs, deactivation for GluN1(G815A) was significantly faster than that for GluN2A(G819A) (Fig. 6e).

One potential explanation for the reduced MOTs and speeding of deactivation rates is that alanine substitutions of the conserved glycines disrupt the stability of the clam-shell closed, agonistbound LBD conformation. To test this possibility, we used cysteine cross-links in the LBD that lock the clam-shell closed (Supplementary Figure 4). The eq. $P_{\text {open }}$ (Supplementary Figure 4b) and MOT (Supplementary Figure 4d) for glycine-toalanine substitutions in either GluN1 or GluN2A was indistinguishable with or without the clam-shell locked close, arguing against any strong effect of the conserved glycine on LBD conformation. We also tested glycine deactivation (Supplementary Figure 5) and it too was significantly faster for both GluN1 $(\mathrm{G} 815 \mathrm{~A})$ and GluN2A(G819A) relative to wild type (Supplementary Figure $5 \mathrm{c}$ ). Finally, desensitization properties for the glycineto-alanine receptors do not appear to contribute strongly to differences in deactivation rates (Fig. 6f).

In summary, alanine substitution of the conserved glycine in either GluN1 or GluN2A attenuates long-lived open states and speeds receptor deactivation, with these effects significantly more prominent in GluN1. These effects are primarily due to this conserved glycine regulating the ion channel conformation as opposed to the LBD conformation.

The conserved glycine maintains the open postures of the M4s. At present there are no open state structures available for NMDARs. To understand how alanine substitutions of the conserved glycines might impact receptor function, we used homology modeling and molecular dynamic (MD) simulations. Based on our previous study ${ }^{35}$, we built a homology model for the GluN1/GluN2B open state using the AMPAR open structure $5 \mathrm{WEO}^{36}$ as template, introduced glycine-to-alanines, and ran simulations for $500 \mathrm{~ns}$ each for wild type, GluN1(G815A)/ GluN2B, and GluN1/GluN2B(G820A) (Supplementary Figure 6 and Fig. 7). Partly due to uncertainty of the wild-type open model, we focused our analysis on the relative difference between glycine-to-alanine mutants and wild type. We refer to this approach as comparative MD simulations.

During the MD simulations, the Ca RMSDs of the entire TMD from the homology model stabilized after about $200 \mathrm{~ns}$ for all constructs (Supplementary Figure 6a). We therefore used the 250-500 ns portion of the MD trajectories to calculate average properties. For wild type the TMD Ca RMSD stabilized at $2.5 \pm$ $0.2 \AA$ (mean value \pm SD) (Supplementary Figure $6 \mathrm{~b}$ ). For both alanine substitutions, the TMD Ca RMSDs increased and, paralleling the impact on MOT, the value was higher for GuN1 $(\mathrm{G} 815 \mathrm{~A})(3.1 \pm 0.1 \AA)$ compared to $\mathrm{GluN} 2 \mathrm{~B}(\mathrm{G} 820 \mathrm{~A})(2.7 \pm$ $0.2 \AA)$. The Ca RMSDs for the TMD core (M1-M3) and the M4 segments showed similar trends (Fig. 7a) though with greater changes over time for the M4 segments (Fig. 7b). Thus, relative to wild type, introducing alanine at the conserved glycine in either
GluN1 or GluN2B alters not only M4 but the entire TMD structure, with these effects stronger for GluN1(G815A).

For wild type, a notable feature was the splaying of the C-termini of the M4 segments, with the hinge or pivot point occurring around the conserved glycine (Fig. 7c, d). In contrast, this splaying was considerably more limited for the GluN1 M4s in GluN1(G815A) (Fig. 7e, f), while a less drastic change was observed for the GluN2 M4s in GluN2B(G820A) (Fig. 7h, i). To quantify this effect, we measured the M4 Ca displacements of GluN1(G815A) (Fig. 7g) and GluN2B(G820A) (Fig. 7j) from wild type. The M4 N-terminal portions of the mutants and wild type remained closely aligned. However, the displacements gradually increased toward the $\mathrm{C}$-termini, where the values were much greater for GluN1 subunits in GluN1(G815A) (Fig. 7g; A subunit, $5.9 \AA$; C, 8.9 $\AA$ ) than for GluN2 subunits in GluN2B(G820A) (Fig. 7j; B subunit, $0.9 \AA$; D, $5.3 \AA$ ). Again, these changes in the positioning of the M4 segments parallel the effect of the alanine substitutions on the stability of the open channel (Fig. 6a-c).

The conserved glycine permits high $\mathrm{Ca}^{2+}$ permeability. Given the differences in C-terminal splaying between the constructs (Fig. 7), we characterized how the glycine-to-alanine mutations might impact the C-terminal portion of the permeation pathway as defined by the M2 pore helix (Fig. $8 \mathrm{a}, \mathrm{b}$ ). For wild type, the average pore radius over the M2 helix is $2.0 \AA$ (minimum at $1.6 \AA$ ) (Fig. 8a, b, gold). In comparison, for GluN1(G815A), the pore is narrower, averaging around $1.6 \AA$ (minimum, $1.2 \AA$ ) (Fig. 8a, right), whereas for GluN2B(G820A), it is unchanged $2.1 \AA$ (minimum at $1.8 \AA$ ) (Fig. 8b, right).

The M2 helix regulates $\mathrm{Ca}^{2+}$ permeability in $\mathrm{NMDARs}^{8}$. We therefore measured relative $\mathrm{Ca}^{2+}$ permeability using changes in reversal potential (Fig. $8 \mathrm{c}, \mathrm{d})^{37}$. Wild-type GluN1/GluN2A, ongoing from 0 to $10 \mathrm{mM} \mathrm{Ca}{ }^{2+}$, showed a change of reversal potential $\left(\Delta E_{\text {rev }}\right)$ of about $10 \mathrm{mV}(9.5 \pm 0.9 \mathrm{mV}, n=7)$, yielding a $P_{\mathrm{Ca}} / P_{\mathrm{Na}}$ of $4(4.1 \pm 0.6)$. For GluN1(G815A), where the M2 pore size was reduced in the MD trajectory, the reversal potential change was significantly reduced $(5.7 \pm 0.5 \mathrm{mV}, n=8)$, corresponding to a decrease in $P_{\mathrm{Ca}} / P_{\mathrm{Na}}$ by about half $(2.0 \pm 0.2, n=8)$. In contrast, GluN1/GluN2A(G819A), where pore size was not altered, $P_{\mathrm{Ca}} / P_{\mathrm{Na}}$ was not significantly changed $(3.7 \pm 0.6, n=7)$.

We also measured $\mathrm{Ca}^{2+}$ permeability for a missense mutation, G815R, at the conserved glycine in GluN1. For this construct, $\mathrm{Ca}^{2+}$ permeability was altered to the same extent as the alanine (Fig. 8d) indicating that electrostatics do not contribute to the role of the GluN1 conserved glycine in modifying $\mathrm{Ca}^{2+}$ permeability.

\section{Discussion}

A key function of NMDARs at synapses is to mediate a timedependent $\mathrm{Ca}^{2+}$ influx that defines synaptic strength and dynamics of integration ${ }^{8,27}$. These features are dependent on the slow deactivation time course of NMDARs in response to transient glutamate released presynaptically and a high $\mathrm{Ca}^{2+}$ permeability. Here, using a combination of functional experiments and MD simulations, our data on clinically relevant mutations show that the C-terminal splaying of the M4 segments, mainly in the GluN1 subunit, regulates both deactivation and high $\mathrm{Ca}^{2+}$ permeability (Fig. 9). These observations have multiple implications for iGluR gating and permeation, including the role of NMDARs in disease and modulation via the C-terminal domain.

The NMDAR open state model was built using homology modeling of an AMPAR open structure ${ }^{36}$ (see Methods). Although this model (Fig. 7c, d) may capture the open state of wild-type NMDARs, we did not test this rigorously in the present 

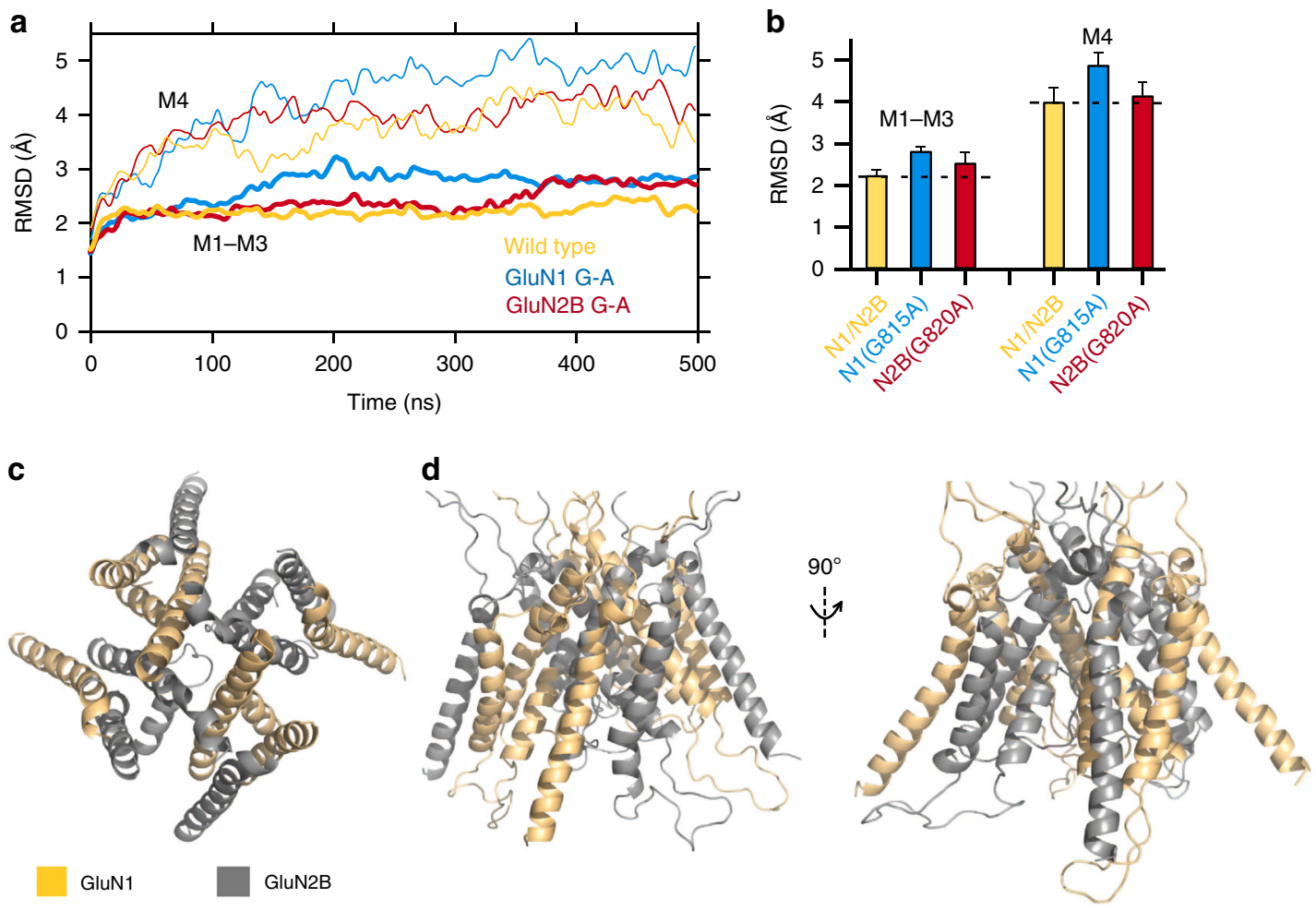

e

$\mathbf{f}$

9

Pair displacement $(\AA ̊)$
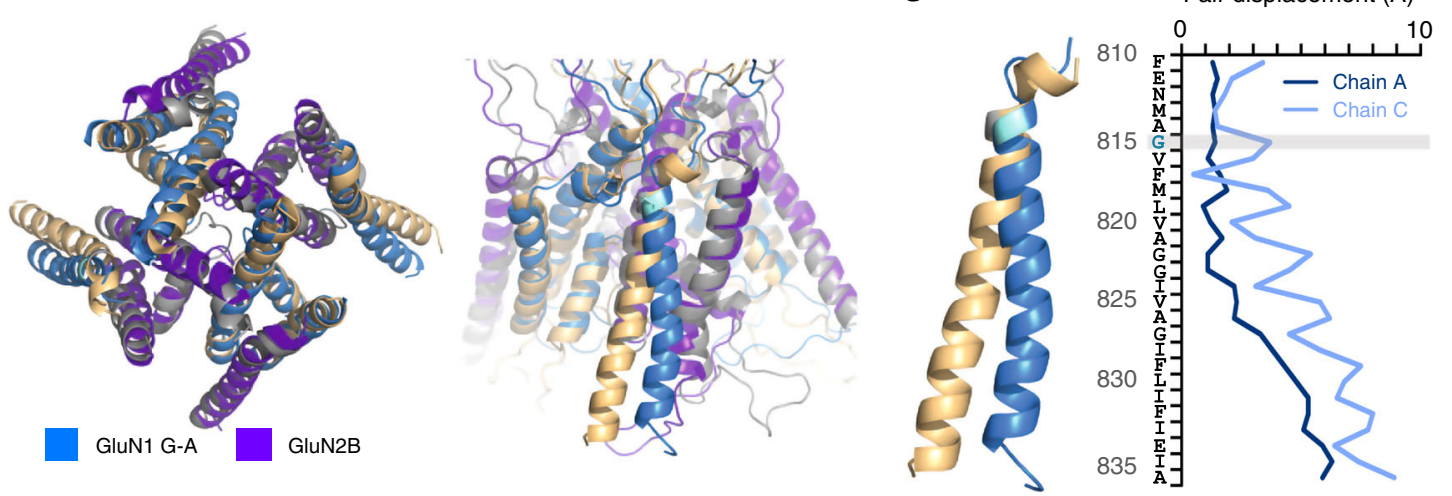

h

i

j
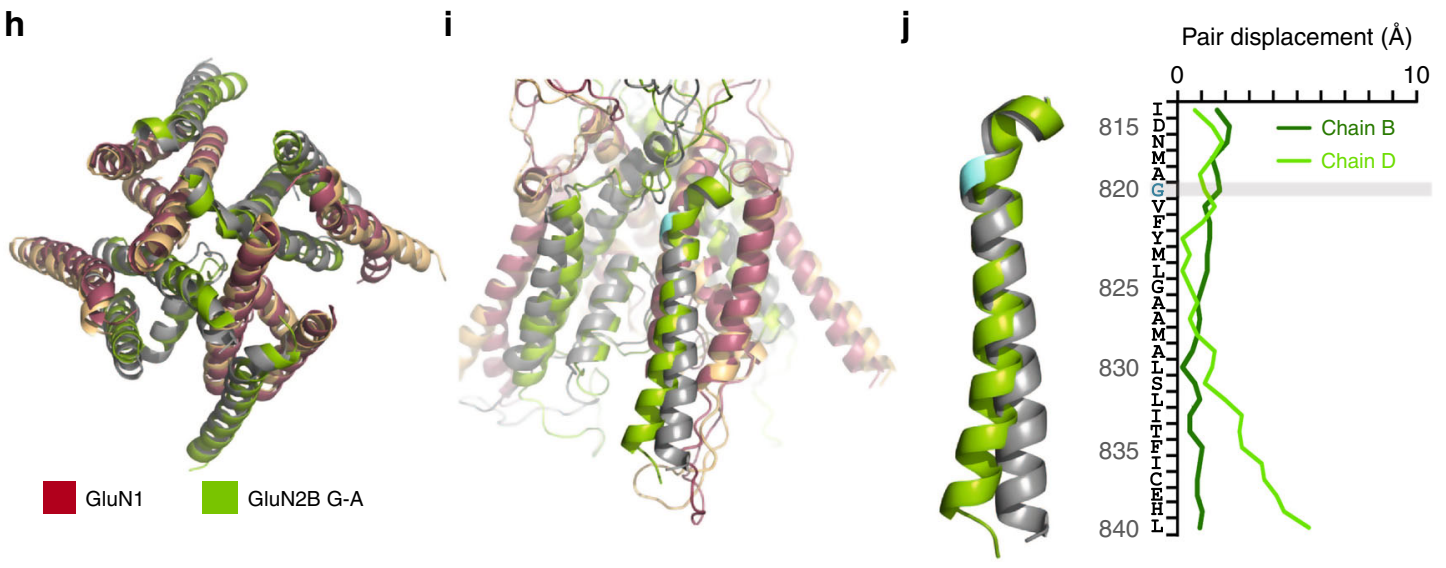

context. Rather, we focused on the relative difference between wild type and constructs containing glycine-to-alanine substitutions either in GluN1 (Fig. 7e-g) or GluN2B (Fig. 7h-j) in an approach that we term comparative MD simulations. Both of these constructs, relative to wild type, showed deviations for all transmembrane segments with these effects consistently stronger for GluN1(G815A) (Fig. 7b and Supplementary Figure 6b). The most notable difference between wild type and the glycine-toalanine constructs was in the M4 segments, as clearly revealed by GluN1(G815A), where the conserved glycine maintains the 
Fig. $7 \mathrm{MD}$ simulations show that constraining the conserved glycine in GluN1 prevents C-terminal expansion of the M4 as observed in wild type. a C $\alpha$ RMSDs of the ion channel cores (M1-M3, thick lines) and M4s (thin lines) as a function of simulation time for open state models: wild-type GluN1/GluN2B, light orange; GluN1(G815A)/GluN2B, light blue; GluN1/GluN2B(G820A), maroon (see Methods). b Average RMSD values (250-500 ns). Error bars are standard deviations. c, d Open state of wild-type GluN1/GluN2B TMD shown either top down (c) or side views (d) of the GluN1 (left) or GluN2B (right) M4s. All displayed structures are snapshots close to the average structures calculated over 250-500 ns. e, $\mathbf{f}$ GluN1(G815A) overlaid on wild type either from top down (e) or with a side view (f). $\mathbf{g}$ Displacements between GluN1(G815A) and wild type for M4 residues in the A and C subunits. $\mathbf{h}, \mathbf{i}$ GluN2B (G820A) overlaid on wild type either from top down (h) or with a side view (i). j Displacements between GluN2B(G820A) and wild type for M4 residues in the $B$ and $D$ subunits

a

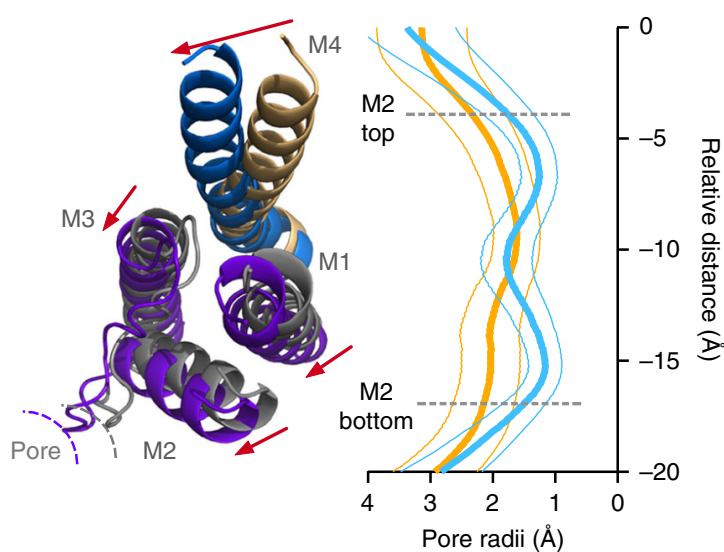

C

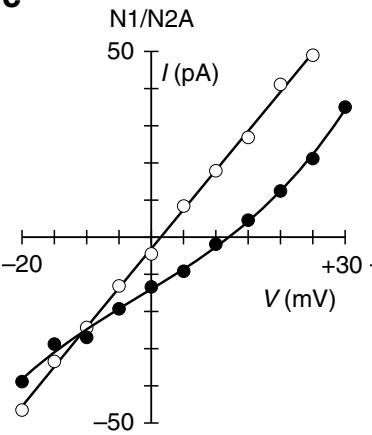

$\mathrm{N} 1(\mathrm{G} 815 \mathrm{~A}) / \mathrm{N} 2 \mathrm{~A}$

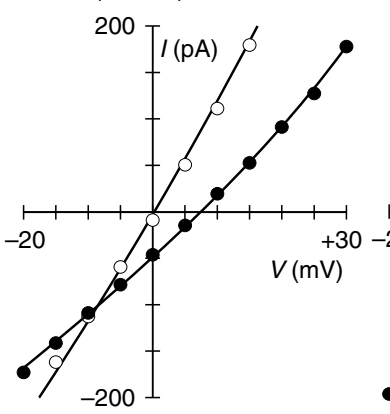

b

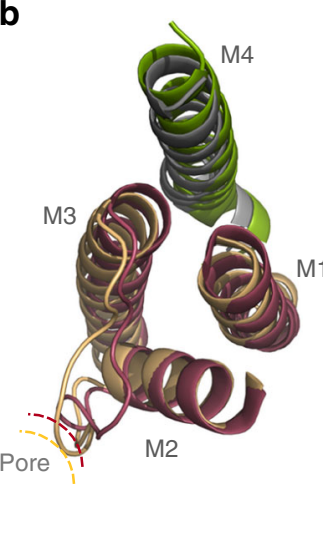

$\mathrm{N} 1 / \mathrm{N} 2 \mathrm{~A}(\mathrm{G} 819 \mathrm{~A})$

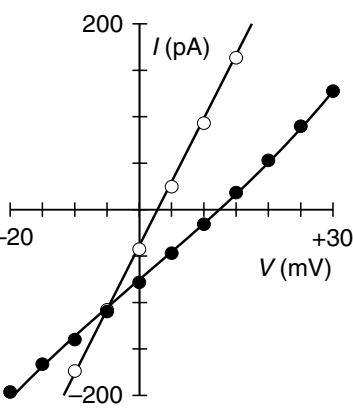

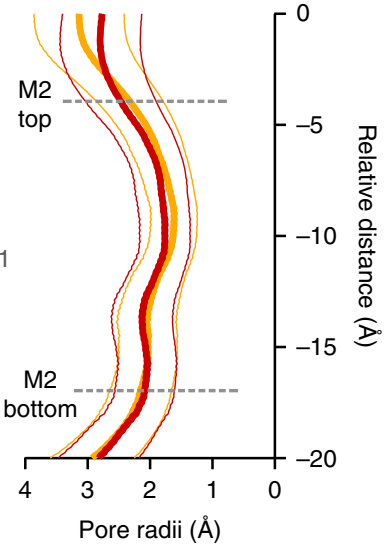

d

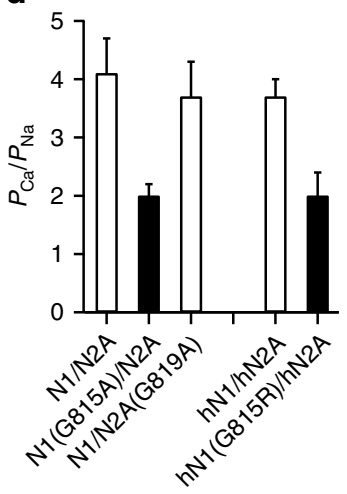

Fig. 8 Constraining the conserved G in GluN1 reduces the dimensions of the selectivity filter and Ca ${ }^{2+}$ permeability. a Left, Cytoplasmic view of model open state structures of wild type and GluN1(G815A). The constrained M4 collapses the adjacent GluN2B ion channel core (M1-M3) including the pore size as defined by the M2 loop. Right, Average pore radius along the channel axis for the M2 pore loop for wild type (gold) and GluN1(G815A) (light blue). ' $\mathrm{O}$ ' references the serine (S) in SYTANLAAF in M3. Thick lines are mean values and thinner lines the error bars, which were calculated using block averages based on a time block of $20 \mathrm{~ns}$. b Same as a except construct is GluN2B(G820A) (maroon), which resulted in a less displacement of the M2 loop. c Current-voltage (IV) relationships in an external solution containing high $\mathrm{Na}^{+}\left(140 \mathrm{mM}\right.$ ) and 0 added $\mathrm{Ca}^{2+}$ (open circles) or $10 \mathrm{mM} \mathrm{Ca}{ }^{2+}$ (solid circles). The $0 \mathrm{Ca}^{2+} \mathrm{IV}$ is the average of that recorded before and after the $10 \mathrm{mM} \mathrm{Ca}{ }^{2+}$ recording. We used changes in reversal potentials $\left(\Delta E_{\text {rev }}\right)$ to calculate $P_{\mathrm{Ca}} / P_{\mathrm{Na}}{ }^{37}$. d Mean $\pm \mathrm{SEM}$ showing relative calcium permeability $\left(P_{\mathrm{Ca}} / P_{\mathrm{Na}}\right)$ calculated from $\Delta E_{\mathrm{rev}} \mathrm{s}$ for rat GluN1/GluN2A $(n=7), \mathrm{GluN1}(\mathrm{G} 815 \mathrm{~A})(n=8)$, or GluN2A(G819A) ( $n=7)$ as well as human GluN1/GluN2A $(n=11)$ and the missense mutation GluN1(G815R) ( $n=7)$. Values either are not (open bars) or are (solids bars) significantly different from each other $(p<0.05$, ANOVA, Tukey)

bottom two-thirds of the helix in an open posture, leaving the upper one-third in close proximity to other TMD elements (Fig. $7 \mathrm{~g}$ ). We assume that the major effect of the glycine-toalanine in GluN1 is to limit this splaying of M4, reducing the stability of long-lived open state (Fig. 9). The glycine-to-alanine substitution in GluN2B also alters the positioning of M4 relative to wild type (Fig. 7j), but this effect is small and may not reflect the major pathway by which the conserved glycine in GluN2 impacts receptor function.

One prediction arising from the comparative MD simulations is that the glycine-to-alanine in GluN1 but not GluN2 restricts the pore diameter of the M2 pore loop (Fig. 8a, b). Supporting this observation is that $\mathrm{Ca}^{2+}$ permeation in GluN1(G815A) but not GluN2A(G819A) was significantly reduced (Fig. 8c, d). This result supports the relative differences in the $\mathrm{MD}$ simulations and also indicates that pore expansion impacts the high $\mathrm{Ca}^{2+}$ influx mediated by NMDARs.

That glycine-to-alanine substitutions at neighboring positions in the M4 segments had no significant effects on receptor gating (Fig. 5) suggest that, rather than merely providing local flexibility to the M4 segments, the conserved glycines play unique roles irreplaceable by any other side chain. In part it may reflect that 


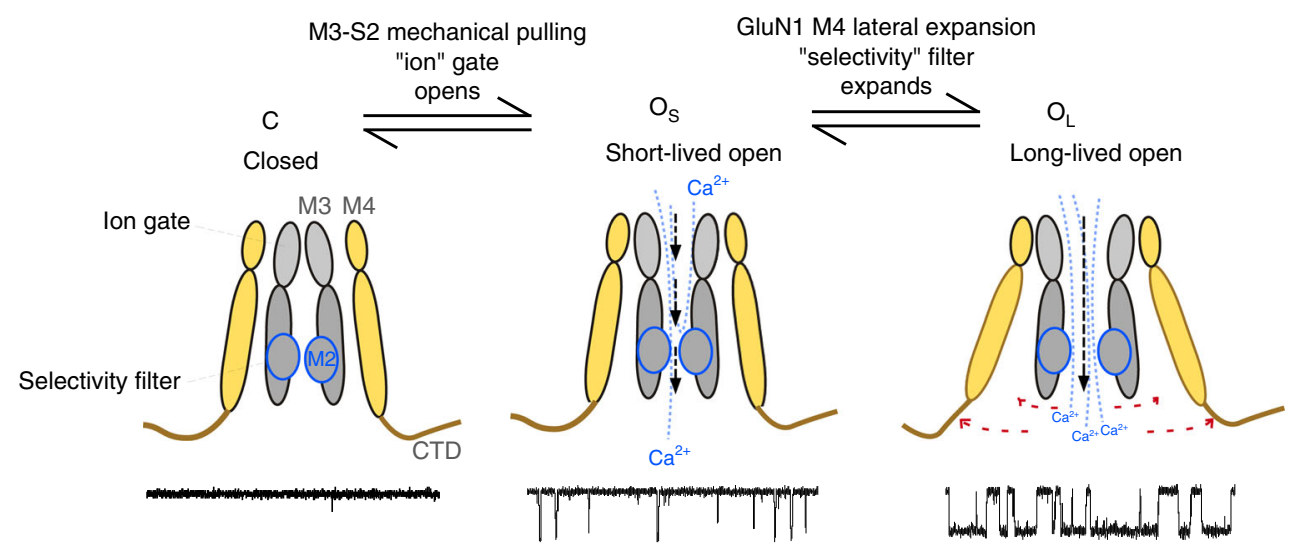

Fig. 9 Expansion of the inner pore permitted mainly by the GluN1 M4 conserved glycine facilitates NMDAR gating and Ca ${ }^{2+}$ permeation. Cartoon of NMDAR pore structure, including the influence of the GluN1 M4 on open state stability and selectivity filter integrity. Subunits are colored light orange (GluN1) and gray (GluN2)

the relative positioning of the glycine in the M4 helix is critical, with structural elements positioned external to the glycine being involved in critical interactions ${ }^{23}$ and those internal to the glycine requiring flexibility ${ }^{16}$. In addition, the open state model suggests a $\mathrm{Ca}$ hydrogen bond that connects the S1-M1 directly to the conserved glycine in the $\mathrm{M} 4^{35}$, which could also alter receptor gating.

We also envision that the M4 is passively, not actively, splaying in response to pore opening. Consistent with this idea, specific side chain interactions of the C-terminal two-thirds of the M4 helix appear unimportant to gating ${ }^{16}$ as would be expected if it acted permissively.

Given the high number of patients identified with mutations in the conserved glycine relative to other M4 transmembrane segment mutations, it is unlikely that mutations at this site are merely epiphenomena. Indeed, such drastic alterations in NMDAR structure and subsequent dysfunction imply a causal relationship. One therefore asks what aspect of this dysfunction plays the dominant role in the pathogenesis of these disorders. Notably, most M4 missense mutations that we measured had reduced channel activity characterized by a destabilized open state (Fig. 3). Though a reduction in excitatory glutamatergic activity leading to epilepsy may seem counterintuitive, it most likely changes the balance of excitation and inhibition in the nervous system.

The most identifiable pathology amongst these mutations is epilepsy. However, a dysfunction common to nearly all M4 missense mutations is intellectual disability (Supplementary Table 1), suggesting that the problem may lie in development. The GluN2B subunit, which carries numerous mutations at the conserved glycine, is most highly expressed before birth ${ }^{27}$. GluN1, being an obligate subunit, is expressed at all stages of development. Interestingly, the absence of any conserved $\mathrm{G}$ mutants in GluN2A, a subunit that is primarily expressed in the adult brain, suggest that pathogenesis of these disorders strongly involves the NMDARs role in the developing brain.

The M4 transmembrane helix in iGluRs is the most peripheral transmembrane segment in the iGluR ion channel, interacts with lipids, and is attached to the regulatory C-terminal domain. Lipids ${ }^{38}$ and post-translational modifications of the Cterminal domain ${ }^{39}$ can modulate ion channel gating and $\mathrm{Ca}^{2+}$ permeation, but how they change receptor function is unknown. We hypothesize that a major pathway by which they act is by regulating the permissiveness of the M4. In addition, agonist binding in NMDARs can induce signaling independent of ion channel opening, including priming them for internalization ${ }^{40}$ and metabotropic actions ${ }^{41}$. Given that the M4s connect the external LBD and the internal CTD and that their displacements would not directly open the channel, the M4 segments could represent the pathway for such transmembrane signaling. Future experiments will be needed to directly test these ideas.

\section{Methods}

Molecular biology and cell culture. All manipulations were made in rat GluN1 (GluN1a) (NCBI Protein database accession no. P35439), GluN2A (Q00959), or GluA2 (P19491) subunits or human GluN1 (hGluN1; Q05586), GluN2A

(hGluN2A; Q12879), or GluN2B (hGluN2B; Q13224) subunits. Unless otherwise noted constructs tested were rat. In all instances, numbering included the signal peptide (GluN1, 18 residues; GluN2A, 19 residues; GluN2B, 26 residues; GluA2, 21 residues). Mutations were generated via QuickChange site-directed mutagenesis (Agilent) with XL1-Blue super-competent cells.

Cell culture and transfection: For details on cell culture and transfection see Amin et al., 2017. Briefly, human embryonic kidney 293 (HEK293) or HEK293T cells were grown in Dulbecco's modified Eagle's medium (DMEM), supplemented with $10 \%$ fetal bovine serum (FBS), for $24 \mathrm{~h}$ prior to transfection. Non-tagged cDNA constructs were co-transfected into HEK293 cells along with a separate pEGFP-Cl vector at a ratio of 4.5:4.5:1 (N1/N2/EGFP for NMDARs) using $\mathrm{X}$-tremeGene HP (Roche). GFP-tagged constructs were transfected at a 1:1 ratio. To improve cell survivability, HEK293 cells were bathed in a media containing the NMDAR competitive antagonist APV $(100 \mu \mathrm{M})$ and $\mathrm{Mg}^{2+}(100 \mu \mathrm{M})$ (single channel experiments) or the transfection mixture was replaced $4 \mathrm{~h}$ after transfection with fresh 5\% FBS-DMEM culture media containing APV $(100 \mu \mathrm{M})$ and $\mathrm{Mg}^{2+}(1 \mathrm{mM})$ (whole-cell and imaging experiments). All experiments were performed $18-48 \mathrm{~h}$ post transfection.

Triheteromeric expression system: NMDARs are obligate heterotetramers typically composed of 2 GluN1 and 2 GluN2 subunits. Co-expression of wild-type GluN1 and GluN2A and a subunit with a mutation [e.g., GluN2A(mutation)] would result in three populations of cell-surface expressed receptors: GluN1/ GluN2A (diheteromeric); GluN1/GluN2A/GluN2A(mutation) (triheteromeric); and GluN1/GluN2A(mutation) (diheteromeric). To restrict surface expression to defined triheteromeric receptors, we used the 'triheteromeric' system developed by the Traynelis lab ${ }^{30}$, which is based on leucine zipper motifs from $G_{A B A}$ receptors and $\mathrm{ER}$ retention/retrieval motifs introduced into the C-terminal domain of NMDAR subunits. All constructs were kindly provided by Dr. Kasper Hansen (University of Montana)

Molecular modeling and simulations. Previously, we constructed an NMDAR open-state model by repacking the transmembrane helices ${ }^{35}$. The model was very similar to the GluA2 open-state structure (Protein Data Bank (PDB) entry 5WEO) 36 , indicating that 5 WEO could be a useful template for modeling the open state of the NMDAR TMD.

Homology modeling: We used homology modeling to build an initial model for the GluN1/GluN2B receptor in the open state (without the disordered C-terminal domain $)^{42}$. We then introduced glycine-to-alanine mutations at the conserved position, producing the N1(G815A)/N2B and N1/N2B(G820A) mutants. For the wild-type open model, we chose PDB entry $5 \mathrm{FXG}^{43}$ as the template for the extracellular amino-terminal domain (ATD) and $\mathrm{LBD}^{35}$, and $5 \mathrm{WEO}^{36}$ as the template for the TMD. The templates were both aligned to the 
closed NMDAR structure $4 \mathrm{TLM}^{44}$. We then used Modeller $9.18^{45}$ for homology modeling, including generation of missing residues.

Our previous study ${ }^{35}$ suggested that interactions between the pre-M1 helix and the extracellular end of the M4 helix are important for stabilizing the open state. Among these interactions are special hydrogen bonds formed with the $\mathrm{Ca}$ atoms of the conserved glycines as donors. Here we introduced such hydrogen bonds into our initial open model, in a 14-ps fine-tuning simulation in vacuum using NAMD 2.11. In this simulation, all Ca atoms were restrained with a force constant of $5 \mathrm{kcal} \mathrm{mol}^{-1} \AA^{-2}$ except for residues K544-Q559 in GluN1 and R540S555 in GluN2B containing the pre-M1 helices. In addition, Ca atoms of G815 in GluN1 and G820 in GluN2B were restrained to a hydrogen-bonding distance of $3.2 \AA$ from backbone carbonyl oxygen atoms of L551 in GluN1 and P547 on GluN2B, respectively, with a force constant of $0.1 \mathrm{kcal} \mathrm{mol}^{-1} \AA^{-2}$. The G815A mutation in GluN1 and G820A mutation in GluN2B were made using VMD ${ }^{46}$ to produce open models for the two mutants.

Comparative molecular dynamics (MD) simulations: Following our previous study ${ }^{35}, \mathrm{MD}$ simulations were carried out for the TMD construct, consisting of residues L541-P670 and R794-A841 in GluN1 and M539-K669 and G799-F845 in GluN2B, truncated from our full-length models. The four LBD-proximal residues of the LBD-TMD linkers (L541-K544, I667-P670, R794-E797 in GluN1 and M537R540, L666-K669, G799-H802 in GluN2B) were restraint to mimic the agonistbound form of the LBD that maintains channel opening. The TMD construct was embedded in a membrane bilayer composed of 222 POPC lipids, and solvated by 25,000 water molecules and $0.15 \mathrm{M} \mathrm{NaCl}$, using CHARMM-GUI ${ }^{47}$. CHARMMGUI's standard 6-step equilibration protocol was followed to equilibrate the system. An additional equilibration step of $12 \mathrm{~ns}$ was used to ramp down the restraints on the $\mathrm{Ca}$ atoms except for the LBD-proximal linker residues. The final production run was $500 \mathrm{~ns}$ with restraints on the LBD-proximal linker Ca remain at $5 \mathrm{kcal} \mathrm{mol}^{-1} \AA^{-2}$.

Starting from the CHARMM-GUI's equilibration step, simulations were performed using AMBER 14 on GPUs ${ }^{48}$ with CHARMM36 force field ${ }^{49}$. The time step was $2 \mathrm{fs}$. Non-bonded interactions were cutoff at $8.0 \AA$, and the particle mesh Ewald method was used for long-range electrostatic interactions ${ }^{48}$ The NPT ensemble was used in simulation, with temperature controlled at $300 \mathrm{~K}$ by Langevin dynamics and pressure controlled at 1 bar by the Berendsen $\operatorname{method}^{50}$.

Analysis: MD trajectories were saved once every 100 ps (5000 frames for each construct). Root-mean-square-deviations (RMSDs) were calculated using $\mathrm{Ca}$ atoms of either all the helical segments (M1-M4) or only M1-M3 in the ion channel core. In the latter case, the RMSD for M4 was also calculated using the structural alignment on M1-M3. The reference for all the RMSD calculations was the homology model. The aligned structures were used to calculate an average structure, which was then used to select a representative snapshot from each MD trajectory. The pore radii along the pore axis were calculated using the HOLE program ${ }^{51}$ on every 10 th of the saved frames.

Macroscopic current recordings. Macroscopic currents in the whole-cell mode or outside-out patches, isolated from HEK293 cells, were recorded at room temperature $\left(20-23^{\circ} \mathrm{C}\right)$ using an EPC-9 or EPC-10 amplifier with Patchmaster software (HEKA Elektronik, Lambrecht, Germany), digitized at $10 \mathrm{kHz}$ and low-pass filtered at $2.8 \mathrm{kHz}(-3 \mathrm{~dB})$ using an 8 pole low pass Bessel filter. Patch microelectrodes were filled with our standard intracellular solution (in $\mathrm{mM}$ ): $140 \mathrm{KCl}, 10$ HEPES, 1 BAPTA, pH 7.2 (KOH). Our standard extracellular solution consisted of (in $\mathrm{mM}$ ): $140 \mathrm{NaCl}, 1 \mathrm{CaCl}_{2}, 10$ HEPES, pH $7.2(\mathrm{NaOH})$. Pipettes had resistances of 2-6 M $\Omega$ when filled with the pipette solution and measured in the standard $\mathrm{Na}^{+}$ external solution. We did not use series resistance compensation nor did we correct for junction potentials. Currents were measured within $15 \mathrm{~min}$ of going whole cell.

External solutions were applied using a piezo-driven double barrel application system. The open tip response (10-90\% rise time) of the application system was between 400 and $600 \mu$ s. For display, NMDAR currents were digitally refiltered at $500 \mathrm{~Hz}$ and resampled at $1 \mathrm{kHz}$.

Rates of deactivation and desensitization: To determine the rates of activation and deactivation, we applied glutamate for $2 \mathrm{~ms}$ at $-70 \mathrm{mV}$ in the whole-cell mode ${ }^{52}$. For activation times, we used the $10-90 \%$ rise time. Deactivation times (weighted $\tau \mathrm{s}$ ) were derived by fitting the decay phase of currents with a doubleexponential function. To determine the extent and rate of desensitization, we applied glutamate at $-70 \mathrm{mV}$ for $2.5 \mathrm{~s}$ in the whole-cell mode. Percent desensitization (\% des) was calculated from the ratio of peak $\left(I_{\text {peak }}\right)$ and steadystate $\left(I_{\text {ss }}\right)$ current amplitudes: \%des $=100 \times\left(1-I_{\text {ss }} / I_{\text {peak }}\right)$. Time constants of desensitization were determined by fitting the decaying phase of currents a doubleexponential function. In some instances when current amplitudes were small, we averaged 3-12 records.

$\mathrm{Ca}^{2+}$ permeability: To quantify $\mathrm{Ca}^{2+}$ permeability, we measured changes in reversal potentials, $\Delta E_{\text {rev }}$, for glutamate-activated currents on replacing a reference solution $\left(140 \mathrm{mM} \mathrm{NaCl}, 10 \mathrm{mM}\right.$ HEPES, 0 added $\left.\mathrm{Ca}^{2+}, \mathrm{pH} 7.2, \mathrm{NaOH}\right)$ with a test solution (the same solution but with added $10 \mathrm{mM} \mathrm{CaCl}_{2}$ ). $\mathrm{Ca}^{2+}$ permeability ratios $\left(P_{\mathrm{Ca}} / P_{\mathrm{Na}}\right)$ were calculated using the Lewis equation ${ }^{37}$. We did not correct for activity coefficients.
Single-channel recordings and analysis. All single-channel recordings were performed in the on-cell configuration at steady state. The pipette solution, which mimicked extracellular agonist conditions, contained (in $\mathrm{mM}$ ): $150 \mathrm{NaCl}, 10$ HEPES, 0.05 EDTA, 1 glutamate, and 0.1 glycine, $\mathrm{pH} 8.0(\mathrm{NaOH})$. The high $\mathrm{pH}$ and EDTA were used to minimize proton and divalent mediated inhibitory effects, respectively ${ }^{28}$. Recording pipettes were pulled from thick-wall borosilicate capillary glass (Sutter Instruments) and fire-polished to final pipette resistances ranging from 5 to $30 \mathrm{M} \Omega$ when measured in the bath solution (with an applied positive pipette pressure of $\sim 200$ mbar). Cells were identified by GFP fluorescence and patched to resistances exceeding $1.5 \mathrm{G} \Omega$. To elicit inward current amplitudes, we held the electrode voltage at $+100 \mathrm{mV}$. Currents were recorded using a patch clamp amplifier (Axopatch 200B; Molecular Devices), filtered at $10 \mathrm{kHz}$ (four-pole Bessel filter), and digitized at $40 \mathrm{kHz}$ (ITC-16 interfaced with PatchMaster). Experiments ran for $\sim 3-20$ min to ensure a significant number of events for analysis.

NMDAR single channel analysis: Analysis of single-channel records was comparable to Talukder and Wollmuth ${ }^{53}$. Data were exported from PatchMaster to QuB. Processed data were idealized using the segmental k-means (SKM) algorithm in $\mathrm{QuB}$ with a dead time of $20 \mu \mathrm{s}$. Closed and open state fits were performed using the maximum interval likelihood (MIL) algorithm in QuB. For most recordings, we measured only single-channel amplitudes, mean closed time (MCT), and mean open time (MOT) as well as equilibrium open probability (eq. $\left.P_{\mathrm{o}}\right)$.

For certain constructs, we explored in more detail the occupancy of different closed and open states. Kinetic models of NMDA receptor gating activation have been proposed to contain $\sim 5$ closed states and 3-4 open states ${ }^{28,29}$ For each individual record, state models with increasing closed (3-6) and open (2-4) states were constructed and fitted to the recordings until loglikelihood (LL) values improved by less than $10 \mathrm{LL}$ units/added state or if the next added state showed $0 \%$ occupancy ${ }^{53}$. To verify single channels in patches, especially for those constructs with a low equilibrium $P_{\mathrm{o}}$, we used statistical approaches $^{31}$

\section{Assaying surface expression using pH-sensitive GFP. HEK293T cells were} imaged $24-48 \mathrm{~h}$ post transfection using a Nikon Ti Eclipse microscope equipped with the Nikon TIRF slider. Excitation used the $488 \mathrm{~nm}$ line from a fiber-coupled argon ion laser (Lasos, Jena, Germany) ${ }^{16}$. The fluorescence emission was collected with a Nikon $\times 601.45 \mathrm{NA}$ oil-immersion TIRF objective and relayed to an iXon DU897 emCCD camera (Andor Technologies, South Windsor, CT). The fluorescence emission was transmitted using a dual band 488/561 TIRF filter cube with a GFP band pass of 525/50 (Chroma, Bellows Falls, VT). Cells were observed at $5 \mathrm{~s}$ intervals with 30-50 exposures collected for each field of view, which contained 1-4 fluorescent cells. Cells were imaged in either a bath solution at $\mathrm{pH}$ 7.4, consisting of (in mM): $140 \mathrm{NaCl}, 10 \mathrm{HEPES}, \mathrm{pH} 7.4(\mathrm{NaOH})$; or a bath solution at $\mathrm{pH}$ 5.5 , consisting of (in $\mathrm{mM}$ ): $140 \mathrm{NaCl}, 302$-( $\mathrm{N}$-morpholino)ethanesulfonic acid (MES), pH $5.5(\mathrm{HCl})$. These bathing solutions were exchanged using a continuous flow perfusion system.

Fluorescence intensity was quantified in Image ${ }^{54}$. Cells were selected for analysis that were at least $50 \%$ isolated from other cells. The fluorescence intensity $(F)$ of a cel of interest was calculated for each frame: $F=F_{\text {cell }}-F_{\text {backgnd }}$ where $F_{\text {cell }}$ is the mean fluorescence of the cell, defined by a polygon circumscribing it, and $F_{\text {backgnd }}$ is the mean fluorescence of an acellular region directly adjacent to the cell of interest. For display and analysis, we normalized fluorescence intensity to a baseline fluorescence $\left(F_{\mathrm{o}}\right)$, which was the mean $F$ just before the solution was changed from $\mathrm{pH} 7.4$ to 5.5 . The change in fluorescence $(\Delta F)$ was defined as $\Delta F=F_{\mathrm{o}}-F_{\text {test }}$, where $F_{\text {test }}$ is the fluorescence intensity taken $15-30 \mathrm{~s}$ after the solution was switched from $\mathrm{pH} 7.4$ to 5.5. We defined 'detectable' surface expression as occurring when the fluorescence intensity decreased rapidly upon switching to $\mathrm{pH} 5.5$ and returned to baseline with a return to $\mathrm{pH}$ 7.4. For some constructs, we detected both positive and negative (no detectable $\Delta F$ ); in these instances, we averaged surface expression only for those cells that were positive.

Statistics. Data analysis was performed using IgorPro, QuB, Excel, and MiniTab 18. All average values are presented as mean \pm SEM. The number of replicates is indicated in the figure legend or in a table associated with the figure. Often, we were only interested in whether outcomes were statistically different from that for wild type. In these instances, we used an unpaired two-tailed Student's $t$-test to test for significant differences. An analysis of variance (ANOVA), followed by the Tukey or Dunnet's test, was used for multiple comparisons. Unless otherwise noted, statistical significance was set at $p<0.05$.

\section{Data availability}

Igor and/or Excel files of all raw data are available upon request.

Received: 16 April 2018 Accepted: 13 August 2018 Published online: 14 September 2018 


\section{References}

1. Noebels, J. Precision physiology and rescue of brain ion channel disorders. J. Gen. Physiol. 149, 533-546 (2017).

2. Kowal, C. et al. Human lupus autoantibodies against NMDA receptors mediate cognitive impairment. Proc. Natl Acad. Sci. USA 103, 19854-19859 (2006).

3. Pleasure, D. Diagnostic and pathogenic significance of glutamate receptor autoantibodies. Arch. Neurol. 65, 589-592 (2008).

4. Burnashev, N. \& Szepetowski, P. NMDA receptor subunit mutations in neurodevelopmental disorders. Curr. Opin. Pharmacol. 20, 73-82 (2015).

5. Yuan, H., Low, C. M., Moody, O. A., Jenkins, A. \& Traynelis, S. F. Ionotropic GABA and glutamate receptor mutations and human neurologic diseases. Mol. Pharmacol. 88, 203-217 (2015).

6. Hardingham, G. E. \& Do, K. Q. Linking early-life NMDAR hypofunction and oxidative stress in schizophrenia pathogenesis. Nat. Rev. Neurosci. 17, 125-134 (2016).

7. Geisheker, M. R. et al. Hotspots of missense mutation identify neurodevelopmental disorder genes and functional domains. Nat. Neurosci. 20, 1043-1051 (2017).

8. Traynelis, S. F. et al. Glutamate receptor ion channels: structure, regulation, and function. Pharmacol. Rev. 62, 405-496 (2010).

9. Swanger, S. A. et al. Mechanistic insight into NMDA receptor dysregulation by rare variants in the GluN2A and GluN2B agonist binding domains. Am. J. Hum. Genet. 99, 1261-1280 (2016).

10. Sobolevsky, A. I., Rosconi, M. P. \& Gouaux, E. X-ray structure, symmetry and mechanism of an AMPA-subtype glutamate receptor. Nature 462, 745-756 (2009).

11. Huettner, J. E. Glutamate receptor pores. J. Physiol. 593, 49-59 (2015).

12. Ren, H., Honse, Y., Karp, B. J., Lipsky, R. H. \& Peoples, R. W. A site in the fourth membrane-associated domain of the N-methyl-D-aspartate receptor regulates desensitization and ion channel gating. J. Biol. Chem. 278, 276-283 (2003).

13. Talukder, I., Borker, P. \& Wollmuth, L. P. Specific sites within the ligandbinding domain and ion channel linkers modulate NMDA receptor gating. J. Neurosci. 30, 11792-11804 (2010).

14. Salussolia, C. L. et al. A eukaryotic specific transmembrane segment is required for tetramerization in AMPA receptors. J. Neurosci. 33, 9840-9845 (2013)

15. Gan, Q., Dai, J., Zhou, H. X. \& Wollmuth, L. P. The transmembrane domain mediates tetramerization of alpha-Amino-3-hydroxy-5-methyl-4isoxazolepropionic acid (AMPA) receptors. J. Biol. Chem. 291, 6595-6606 (2016).

16. Amin, J. B. et al. Divergent roles of a peripheral transmembrane segment in AMPA and NMDA receptors. J. Gen. Physiol. 149, 661-680 (2017).

17. Chen, W. et al. Functional evaluation of a de novo GRIN2A mutation identified in a patient with profound global developmental delay and refractory epilepsy. Mol. Pharmacol. 91, 317-330 (2017).

18. Partridge, A. W., Therien, A. G. \& Deber, C. M. Missense mutations in transmembrane domains of proteins: phenotypic propensity of polar residues for human disease. Proteins 54, 648-656 (2004).

19. Molnar, J., Szakacs, G. \& Tusnady, G. E. Characterization of diseaseassociated mutations in human transmembrane proteins. PLOS ONE 11, $\mathrm{e} 0151760$ (2016)

20. Moore, D. T., Berger, B. W. \& DeGrado, W. F. Protein-protein interactions in the membrane: sequence, structural, and biological motifs. Structure 16, 991-1001 (2008).

21. Jiang, Y. et al. The open pore conformation of potassium channels. Nature 417, 523-526 (2002)

22. Liu, S. et al. A rare variant identified within the GluN2B C-terminus in a patient with autism affects NMDA receptor surface expression and spine density. J. Neurosci. 37, 4093-4102 (2017).

23. Yuan, H. et al. Functional analysis of a de novo GRIN2A missense mutation associated with early-onset epileptic encephalopathy. Nat. Commun. 5, 3251 (2014).

24. Fedele, L. et al. Disease-associated missense mutations in GluN2B subunit alter NMDA receptor ligand binding and ion channel properties. Nat. Commun. 9, 957 (2018).

25. Lester, R. A., Clements, J. D., Westbrook, G. L. \& Jahr, C. E. Channel kinetics determine the time course of NMDA receptor-mediated synaptic currents. Nature 346, 565-567 (1990).

26. Vicini, S. et al. Functional and pharmacological differences between recombinant N-methyl- D-aspartate receptors. J. Neurophysiol. 79, 555-566 (1998).

27. Paoletti, P., Bellone, C. \& Zhou, Q. NMDA receptor subunit diversity: impact on receptor properties, synaptic plasticity and disease. Nat. Rev. Neurosci. 14 383-400 (2013).

28. Popescu, G. \& Auerbach, A. Modal gating of NMDA receptors and the shape of their synaptic response. Nat. Neurosci. 6, 476-483 (2003).

29. Banke, T. G. \& Traynelis, S. F. Activation of NR1/NR2B NMDA receptors. Nat. Neurosci. 6, 144-152 (2003).
30. Hansen, K. B., Ogden, K. K., Yuan, H. \& Traynelis, S. F. Distinct functional and pharmacological properties of Triheteromeric GluN1/GluN2A/GluN2B NMDA receptors. Neuron 81, 1084-1096 (2014).

31. Kazi, R. et al. Asynchronous movements prior to pore opening in NMDA receptors. J. Neurosci. 33, 12052-12066 (2013).

32. Ding, S., Ingleby, L., Ahern, C. A. \& Horn, R. Investigating the putative glycine hinge in Shaker potassium channel. J. Gen. Physiol. 126, 213-226 (2005).

33. Kussius, C. L. \& Popescu, G. K. Kinetic basis of partial agonism at NMDA receptors. Nat. Neurosci. 12, 1114-1120 (2009).

34. Zhang, W., Howe, J. R. \& Popescu, G. K. Distinct gating modes determine the biphasic relaxation of NMDA receptor currents. Nat. Neurosci. 11, 1373-1375 (2008).

35. Pang, X. \& Zhou, H. X. Structural modeling for the open state of an NMDA receptor. J. Struct. Biol. 200, 369-375 (2017).

36. Twomey, E. C., Yelshanskaya, M. V., Grassucci, R. A., Frank, J. \& Sobolevsky, A. I. Channel opening and gating mechanism in AMPA-subtype glutamate receptors. Nature 549, 60-65 (2017)

37. Jatzke, C., Watanabe, J. \& Wollmuth, L. P. Voltage and concentration dependence of $\mathrm{Ca}^{2+}$ permeability in recombinant glutamate receptor subtypes. J. Physiol. 538, 25-39 (2002).

38. Korinek, M. et al. Cholesterol modulates open probability and desensitization of NMDA receptors. J. Physiol. 593, 2279-2293 (2015).

39. Aman, T. K., Maki, B. A., Ruffino, T. J., Kasperek, E. M. \& Popescu, G. K. Separate intramolecular targets for protein kinase A control N-Methyl-daspartate receptor gating and $\mathrm{Ca}^{2+}$ permeability. J. Biol. Chem. 289, 18805-18817 (2014)

40. Nong, Y. et al. Glycine binding primes NMDA receptor internalization. Nature 422, 302-307 (2003)

41. Nabavi, S. et al. Metabotropic NMDA receptor function is required for NMDA receptor-dependent long-term depression. Proc. Natl Acad. Sci. USA 110, 4027-4032 (2013).

42. Dai, J. \& Zhou, H. X. An NMDA receptor gating mechanism developed from MD simulations reveals molecular details underlying subunit-specific contributions. Biophys. J. 104, 2170-2181 (2013).

43. Tajima, N. et al. Activation of NMDA receptors and the mechanism of inhibition by ifenprodil. Nature 534, 63-68 (2016).

44. Lee, C. H. et al. NMDA receptor structures reveal subunit arrangement and pore architecture. Nature 511, 191-197 (2014).

45. Eswar, N. et al. Comparative protein structure modeling using MODELLER Curr. Protoc. Protein Sci. (2007). Chapter 2: Unit 2-9.

46. Humphrey, W., Dalke, A. \& Schulten, K. VMD: visual molecular dynamics. J. Mol. Graph. 14, 33-38 (1996). 27-38.

47. Jo, S., Kim, T., Iyer, V. G. \& Im, W. CHARMM-GUI: a web-based graphical user interface for CHARMM. J. Comput. Chem. 29, 1859-1865 (2008).

48. Salomon-Ferrer, R., Gotz, A. W., Poole, D., Le Grand, S. \& Walker, R. C. Routine microsecond molecular dynamics simulations with AMBER on GPUs. 2. Explicit Solvent Particle Mesh Ewald. J. Chem. Theory Comput. 9, 3878-3888 (2013).

49. Klauda, J. B. et al. Update of the CHARMM all-atom additive force field for lipids: validation on six lipid types. J. Phys. Chem. B 114, 7830-7843 (2010).

50. van Gunsteren, W. F. \& Berendsen, H. J. Computer simulation as a tool for tracing the conformational differences between proteins in solution and in the crystalline state. J. Mol. Biol. 176, 559-564 (1984).

51. Smart, O. S., Neduvelil, J. G., Wang, X., Wallace, B. A. \& Sansom, M. S. HOLE: a program for the analysis of the pore dimensions of ion channel structural models. J. Mol. Graph. 14, 354-360 (1996). 376.

52. Kazi, R., Dai, J., Sweeney, C., Zhou, H. X. \& Wollmuth, L. P. Mechanical coupling maintains the fidelity of NMDA receptor-mediated currents. Nat. Neurosci. 17, 914-922 (2014)

53. Talukder, I. \& Wollmuth, L. P. Local constraints in either the GluN1 or GluN2 subunit equally impair NMDA receptor pore opening. J. Gen. Physiol. 138, 179-194 (2011).

54. Schneider, C. A., Rasband, W. S. \& Eliceiri, K. W. NIH Image to ImageJ: 25 years of image analysis. Nat. Methods 9, 671-675 (2012).

\section{Acknowledgements}

We thank Dr. Rashek Kazi and Kelvin Chan for helpful discussions and/or comments on the manuscript, Drs. Stephen Traynelis and Hongjie Yuan (Emory University) for generously sharing human NMDAR subunits, Dr. Kasper Hansen (University of Montana) for supplying triheteromeric constructs, and Gabrielle Moody for assisting with Supplementary Table 1. Dr. Steve Smith (Stony Brook) provided invaluable insights about the importance of glycines in transmembrane interactions. This work was sup ported by the NIH Grants R01 NS088479 (L.P.W.), including a minority supplement (J.B.A.), and R35 GM118091 (H.-X.Z.).

\section{Author contributions}

J.B.A., H.-X.Z., and L.P.W. designed research; J.B.A., A.G., and L.P.W. carried out and/or analyzed the functional experiments; X.L. and H.-X.Z., performed the computational 
studies, including modeling and molecular dynamic simulations; J.B.A., X.L., H.-X. Z., and L.P.W. wrote the paper.

\section{Additional information}

Supplementary Information accompanies this paper at https://doi.org/10.1038/s41467018-06145-w.

Competing interests: The authors declare no competing interests.

Reprints and permission information is available online at http://npg.nature.com/ reprintsandpermissions/

Publisher's note: Springer Nature remains neutral with regard to jurisdictional claims in published maps and institutional affiliations. (c) (i) Open Access This article is licensed under a Creative Commons Attribution 4.0 International License, which permits use, sharing, adaptation, distribution and reproduction in any medium or format, as long as you give appropriate credit to the original author(s) and the source, provide a link to the Creative Commons license, and indicate if changes were made. The images or other third party material in this article are included in the article's Creative Commons license, unless indicated otherwise in a credit line to the material. If material is not included in the article's Creative Commons license and your intended use is not permitted by statutory regulation or exceeds the permitted use, you will need to obtain permission directly from the copyright holder. To view a copy of this license, visit http://creativecommons.org/ licenses/by/4.0/.

(C) The Author(s) 2018 